Suresha Suraiah Palaiah, Hussain Basha*, and Gudala Janardhana Reddy*

\title{
Magnetized couple stress fluid flow past a vertical cylinder under thermal radiation and viscous dissipation effects
}

https://doi.org/10.1515/nleng-2021-0027

Received Apr 22, 2021; accepted Sep 16, 2021.

\begin{abstract}
Contemporary investigation studies the silent features of the dissipative free convection couple stress fluid flow over a cylinder under the action of magnetic field, thermal radiation and porous medium with chemical reaction effect. Present two-dimensional viscous incompressible physical model is designed based on the considered flow geometry. Present physical problem gives the highly complicated nonlinear coupled partial differential equations (PDE's) which are not amenable to any of the known techniques. Thus, unconditionally stable, most accurate and speed converging with flexible finite difference implicit technique is utilized to simplify the dimensionless flow field equations. It is apparent from the current results that; the velocity profiles are diminished with enhancing values of magnetic field. Temperature profile increases with enhancing values of thermal radiation parameter. Velocity contours deviates away from the wall with enhancing magnetic parameter. Also, the effects of magnetic field, porous medium, thermal radiation, chemical reaction, buoyancy ratio parameter and Eckert number on couple stress flow velocity, temperature, and concentration profiles are studied. However, the present study has good number of applications in the various fields of engineering such as; polymer processing, solidification of liquid crystals, colloidal solutions, synovial joints, geophysics, chemical engineering, astrophysics and nuclear reactors etc. Finally, the current solutions are validated with the available results in the literature review and found to be in good agreement.
\end{abstract}

Keywords: couple stress fluid, vertical cylinder, chemical reaction, radiation effect, viscous dissipation

Suresha Suraiah Palaiah, Department of Mathematics, Central University of Karnataka, Kalaburagi 585367, India

*Corresponding Author: Hussain Basha, Gudala Janardhana Reddy, Department of Mathematics, Central University of Karnataka, Kalaburagi 585367, India, E-mail: hussainbmaths@gmail.com, gjr@cuk.ac.in

\section{Introduction}

Incompressible couple stress fluid flow obeying Prandtl's theory with distinct effects about a vertical cylinder kept in a porous medium is a significant study area related to many of the manufacturing process. The non-Newtonian fluids have rich set of biomedical and industrial applications in the field of science and engineering. Numerous non-Newtonian fluids are available in nature which has significant number of applications in the various fields of science and engineering. Particularly, the time-dependent couple stress fluid have important applications in numerous processes that are recognized in the industry like the extrusion of polymer fluids, solidification of liquid crystals, and colloidal solutions. Application of couple stress fluid is fairly found in synovial joints (shoulder, hip, knee, and ankle), geophysics, chemical engineering, and astrophysics. Based on the literature review it is observed that, many researchers modelled the synovial fluids as couple stress fluid and they investigated the thermodynamic behaviour. In the last few decades, the flow of couple stress liquid around a plate and cylinder with different physical effects has acknowledged the consideration of various researchers and engineers.

Soundalgekar [1] investigated the natural convection flow over an infinite vertical plate by considering Stokes problem in accordance with the mass transfer applications. The action of variable temperature on fluid flow about a moving plate was investigated by Murty and Soundalgekar [2]. Soundalgekar and Takher [3] employed the series solution for magnetized free convection flow past an infinite vertical plate under the action of uniform transverse magnetic field with dissipation effect. Their investigation demonstrates that, the rising magnetic field parameter and the Prandtl number enhances the dissipation effects in the flow regime. Ganesan and Rani [4, 5] were disclosed the transient natural convection fluid flow with heat and mass transfer process along the vertical cylinder by considering MHD effects. Fathia et al. [6] studied the dissipative unsteady natural convective viscous fluid flow with concentration diffusion about an infinite 
vertical plate. Rani [7] has examined the time-dependent buoyancy motivated flow with thermal and species transfer effects over a vertical cylinder. Unsteady magnetized natural convective viscous dissipative fluid flow about a vertical plate was studied by Singh et al. [8] and they visualized that, the rising magnetic field raises the temperature field.

Palani [9] studied the viscous dissipative fluid flow along a vertical plate with variable temperature field. The research revealed that, the increasing dissipation effect results in the rise of temperature. Rani and Kim studied the time-dependent natural convection flow over a vertical cylinder with variable viscosity and thermal conductivity along with temperature dependent viscosity effect $[10,11]$. Rani and Kim examined the unsteady natural convection flow about an isothermal cylinder with Dufour and Soret effects [12]. Magnetised fluid flow with diffusion of concentration field about a vertical plate was studied by Palani and Srikanth [13]. They found that, the rising buoyancy force significantly raises the velocity field. The effects of variable viscosity and heat conduction on velocity, temperature and Nusselt number on vertical geometry were studied by Palani and Kim [14]. Rani and Kim studied the time-dependent free convective micro-polar fluid flow about a cylinder with heat and mass transfer [15]. Rani et $a l$. numerically examined the chemically reactive couple stress fluid flow about a vertical cylinder [16, 17]. In the same direction, the problem of fluid moment about a moving cylinder with magnetic and thermal radiation impacts was discussed by Loganathan et al. [18].

Neog and Das investigated the unsteady free convective magnetised fluid flow past a plate with varying temperature by employing Laplace transform method [19]. Rani et al. studied the buoyancy motivated unsteady couple stress fluid flow over a cylinder under the action of Prandtl number [20]. Bella and Naikoti inspected the impacts of dissipation and thermal radiative on timedependent natural convective magnetized chemically reactive thermal and mass moment about a plumb plate using FEM method [21]. The application of viscosity on free convection flow of air around a vertical cylinder was studied by Hossain et al. [22]. Loganathan and Sivapoornapriya studied the free convective species concentration transfer past an infinite plate which is kept in porous medium [23]. Their analysis shows that, the rising permeability parameter enhances the velocity field and rising Prandtl number diminishes the thermal field.

Reddy and Raju analyzed the radiation and chemical reaction effects on unsteady magnetized mixed convective flow around a vertical porous plate [24]. Palani et $a l$. disclosed the impacts of MHD and viscous dissipation on buoyancy motivated fluid moment about a plate with varying thermal field [25]. Loganathan and Divya numerically discussed the effects of chemical reaction and dissipation on Newtonian fluid past a cylinder [26]. Reddy studied the impact thermal radiation and chemical application on time-dependent magnetized free convective parabolic fluid flow over isothermal vertical plate with viscous dissipation effect [27]. Basha et al. studied the impacts of free convection on supercritical water flow past a vertical using Redlich-Kwong equation of state approach [28]. Basha et al. studied the chemically reactive unsteady buoyancy motivated couple stress fluid flow about a vertical plate [29]. Recently, Shekar and Kishan studied the impact of thermal radiation on natural convection thermal transfer in a square cavity filled with nanofluid under the influence of saturated porous medium with the different nanoparticles [30]. It is found from their investigation that, the heat transfer increases with increase in Rayleigh number. Haritha et al. investigated the impact of magnetic and viscous dissipation on free convection heat transfer process in a square cavity filled with nanofluid under the effect of saturated porous medium with the different nanoparticles [31]. It is observed from their investigation that, the enhancing magnetic number increases the flow field. Balla et al. numerically studied the effect of chemical reaction on bioconvective flow in a porous square cavity containing oxytactic microorganism [32]. Further, the bio-convection thermal and flow behavior is studied under the impact of Darcy model. It is recorded from their investigation that, chemical reaction increases the flow cell and causes the splitting of the cell in the fluid medium.

Acharya et al. studied the impact of thermal radiation on laminar free convective two-dimensional couple stress fluid flow over a stretching cylinder under magnetic field [33]. It is recorded from their study that, the increasing magnetic number increases the entropy generation process. Basha et al. investigated supercritical free convection in couple stress and Newtonian fluids under the influence EOS approach [34]. It is found from their investigation that, the supercritical fluid is sensible to thermal changes in the fluid medium. Das et al. [35] numerically investigated the importance of Joule heating, viscous dissipation, magnetic field and slip condition on the two-dimensional flow of an electrically conducting couple stress fluid induced by an exponentially elongating sheet kept in a porous medium. It is recorded from their study that, the increasing radiation number increases the thermal field in the flow regime. Ibrahim and Gadisa [36] studied the double stratified mixed convection flow of couple stress nanofluid about an inclined elongating cylinder under the impact of Cattaneo-Christov heat and mass flux model. It is found 
from their study that, the increasing Reynolds number increases the concentration distribution in the flow field. The detailed literature on couple stress fluid can be found in [37-40].

By observing the above published results, the combined effects of chemical reaction with radiation, dissipation and porous medium on incompressible unsteady buoyancy driven couple stress fluid flow around a vertical cylinder has obtained rare consideration in the literature. In the current work, authors made an attempt to examine the behaviour of time-dependent couple stress fluid flow about a cylinder with magnetic force, porous medium, thermal radiation and chemical reaction. However, yet the exact solution is not available for the current physical problem with above mentioned physical effects. The governing highly nonlinear physical system of flowfield equations are simplified by employing the unconditionally stable finite difference scheme.

\section{Mathematical formulation and governing equations}

Transient natural convective magnetised incompressible thermally radiative viscous couple stress fluid about a vertical cylinder with viscous dissipation and chemical reaction impacts under the influence of porous medium is studied. To define the problem well, a rectangular region of geometry is considered in which $x$-axis is measured along the axial path and $r$-coordinate is taken normal to cylinder. The schematic representation of the physical problem shown in Figure 1. The free stream thermal field $\left(T_{\infty}^{\prime}\right)$ and species diffusion field $\left(C_{\infty}^{\prime}\right)$ are expected to be same as the thermal and mass diffusion of the geometry which is maintained at the time $t^{\prime}=0$. As time starts $t^{\prime}>0$, the thermal field and concentration diffusion raised to $T_{w}^{\prime}\left(>T_{\infty}^{\prime}\right)$ and $C_{w}^{\prime}\left(>C_{\infty}^{\prime}\right)$ and this is same for all time $t^{\prime}>0$. With this thermal and mass diffusion differences within the boundary layer in the vicinity of the flow configuration density changes occurs and that in turn interacts with the gravity field and produces the free convective couple stress fluid flow about a cylinder. Based on the fluid flow and geometry, the governing two-dimensional nonlinear coupled equations of the present problem are described below with Boussinesq's approximation [10-12, 15-17].

\section{Continuity equation:}

$$
\frac{\partial(r u)}{\partial x}+\frac{\partial(r v)}{\partial r}=0
$$

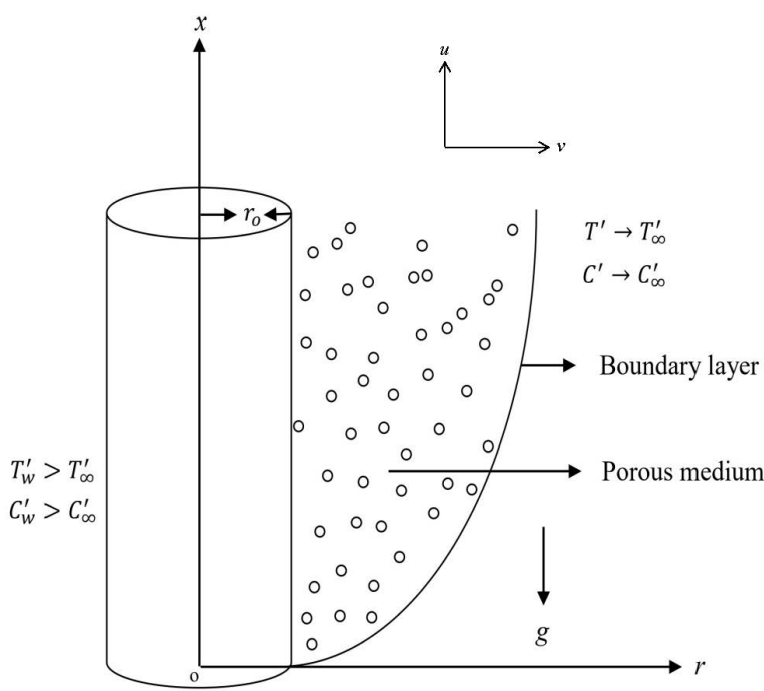

Figure 1: Schematic representation of the problem.

\section{Momentum equation:}

$$
\begin{aligned}
& \rho\left(\frac{\partial u}{\partial t^{\prime}}+u \frac{\partial u}{\partial x}+v \frac{\partial u}{\partial r}\right)=\rho g \beta_{T}\left(T^{\prime}-T_{\infty}^{\prime}\right)+\rho g \beta_{C}\left(C^{\prime}-C_{\infty}^{\prime}\right) \\
& +\frac{1}{r} \frac{\partial}{\partial r}\left(\mu r \frac{\partial u}{\partial r}\right)-\frac{\mu}{K_{1}^{\prime}} u-\eta \nabla^{4} u-\sigma B_{0}^{2} u
\end{aligned}
$$

Here $\eta$ is the constant related to couple stress liquid flow.

\section{Energy equation:}

$$
\begin{aligned}
& \left(\frac{\partial T^{\prime}}{\partial t^{\prime}}+u \frac{\partial T^{\prime}}{\partial x}+v \frac{\partial T^{\prime}}{\partial r}\right)=\frac{\alpha}{r} \frac{\partial}{\partial r}\left(r \frac{\partial T^{\prime}}{\partial r}\right)+\frac{\mu}{\rho C_{p}}\left(\frac{\partial u}{\partial r}\right)^{2} \\
& -\frac{1}{\rho C_{p}} \frac{1}{r} \frac{\partial}{\partial r}\left(r q_{r}\right)
\end{aligned}
$$

\section{Concentration equation:}

$$
\left(\frac{\partial C^{\prime}}{\partial t^{\prime}}+u \frac{\partial C^{\prime}}{\partial x}+v \frac{\partial C^{\prime}}{\partial r}\right)=\frac{D}{r} \frac{\partial}{\partial r}\left(r \frac{\partial C^{\prime}}{\partial r}\right)-K_{r}^{\prime}\left(C^{\prime}-C_{\infty}^{\prime}\right)
$$

Based on the Rosseland theory of thermal radiation [41], the radiative heat flux $\left(q_{r}\right)$ can be approximated as:

$$
q_{r}=-\frac{4 \sigma_{s}}{3 k^{\star}} \frac{\partial T^{\prime 4}}{\partial r}
$$

Stefan-Boltzmann constant is $\sigma_{s}$ and $k^{\star}$ is the mean absorption coefficient. If difference of the temperature within the flow is too small, the term $T^{\prime 4}$ can be written as a linear temperature function, then $T^{\prime 4}$ can be written by utilizing Taylor's expansion about $T_{\infty}^{\prime}$ with ignoring terms of greater order is given by:

$$
T^{\prime 4} \cong 4 T_{\infty}^{\prime 3}-3 T_{\infty}^{\prime 4}
$$


Using equations (5) and (6), equation (3) reduces to

$$
\left(\frac{\partial T^{\prime}}{\partial t^{\prime}}+u \frac{\partial T^{\prime}}{\partial x}+v \frac{\partial T^{\prime}}{\partial r}\right)=\frac{\alpha}{r} \frac{\partial}{\partial r}\left(r \frac{\partial T^{\prime}}{\partial r}\right)+\frac{\mu}{\rho C_{p}}\left(\frac{\partial u}{\partial r}\right)^{2}+\frac{16 \sigma_{s} T_{\infty}^{\prime 3}}{3 \rho C_{p} k^{\star}} \frac{1}{r} \frac{\partial}{\partial r}\left(r \frac{\partial T^{\prime}}{\partial r}\right)
$$

Stokes [16, 17] given the two conditions for couple stress liquid moment in the boundary layer regime. However, second condition is employed for simplifying the current problem. Thus, the required boundary conditions of the current physical problem are [10-12, 15-17, 20, 34]:

$$
t^{\prime} \leq 0: \quad u=0, v=0, \quad T^{\prime}=T_{\infty}^{\prime}, C^{\prime}=C_{\infty}^{\prime}
$$

for all $x \& r$

$$
t^{\prime}>0: \quad u=0, v=0, T^{\prime}=T_{w}^{\prime}, C^{\prime}=C_{w}^{\prime} \text { at } r=r_{0}
$$

$u=0, \quad v=0, \quad T^{\prime}=T_{\infty}^{\prime}, C^{\prime}=C_{\infty}^{\prime}$ at $x=0$

$T^{\prime} \rightarrow T_{\infty}^{\prime}, C^{\prime} \rightarrow C_{\infty}^{\prime}, u \rightarrow 0, v \rightarrow 0$ as $r \rightarrow \infty$

and

$$
\frac{\partial u}{\partial r}=\frac{\partial v}{\partial x} \text { at } r=r_{0} \text { and } r \rightarrow \infty
$$

Utilized non-dimensional variables and parameters are as follows:

$$
\begin{gathered}
X=G r_{T}^{-1} \frac{x}{r_{0}}, \quad R=\frac{r}{r_{0}}, \quad U=G r_{T}^{-1} \frac{u r_{0}}{v}, \quad V=\frac{v r_{0}}{v}, \quad t=\frac{v t^{\prime}}{r_{0}^{2}}, \\
T=\frac{T^{\prime}-T_{\infty}^{\prime}}{T_{w}^{\prime}-T_{\infty}^{\prime}}, \quad C=\frac{C^{\prime}-C_{\infty}^{\prime}}{C_{w}^{\prime}-C_{\infty}^{\prime}}, \\
G r_{T}=\frac{g \beta_{T} r_{0}^{3}\left(T_{w}^{\prime}-T_{\infty}^{\prime}\right)}{v^{2}}, G r_{C}=\frac{g \beta_{C} r_{0}^{3}\left(C_{w}^{\prime}-C_{\infty}^{\prime}\right)}{v^{2}}, \\
P r=\frac{v}{a}, r_{0}=\left(\frac{\eta}{\mu}\right)^{1 / 2}, S c=\frac{v}{D}, B u=\frac{G r_{C}}{G r_{T}}, \\
M=\frac{\sigma B_{0}^{2} r_{0}^{2}}{\rho v}, \quad K 1=\frac{k_{1}^{\prime}}{r_{0}^{2}}, K r=\frac{K_{r}^{\prime} r_{0}^{2}}{v}, N r=\frac{k^{\star} k}{4 \sigma_{s} T_{\infty}^{3}}, \\
E c=\frac{G r_{T}^{2} v^{2}}{C_{p} r_{0}^{2}\left(T_{w}^{\prime}-T_{\infty}^{\prime}\right)} .
\end{gathered}
$$

By introducing the above dimensionless quantities in the Eqs. (1)-(4) and (7), the reduced non-dimensional governing equations are obtained:

$$
\begin{gathered}
\frac{\partial U}{\partial X}+\frac{\partial V}{\partial R}+\frac{V}{R}=0 \\
\left(\frac{\partial U}{\partial t}+U \frac{\partial U}{\partial X}+V \frac{\partial U}{\partial R}\right)=T+B u C-\frac{U}{K 1}-M U+\left(1+\frac{1}{R^{2}}\right) \frac{\partial^{2} U}{\partial R^{2}}+\left(\frac{1}{R}-\frac{1}{R^{3}}\right) \frac{\partial U}{\partial R}-\frac{2}{R} \frac{\partial^{3} U}{\partial R^{3}}-\frac{\partial^{4} U}{\partial R^{4}} \\
\left(\frac{\partial T}{\partial t}+U \frac{\partial T}{\partial X}+V \frac{\partial T}{\partial R}\right)=\frac{1}{P r}\left(1+\frac{4}{3 N r}\right)\left(\frac{\partial^{2} T}{\partial R^{2}}+\frac{1}{R} \frac{\partial T}{\partial R}\right)+E c\left(\frac{\partial U}{\partial R}\right)^{2} \\
\left(\frac{\partial C}{\partial t}+U \frac{\partial C}{\partial X}+V \frac{\partial C}{\partial R}\right)=\frac{1}{S c}\left(\frac{\partial^{2} C}{\partial R^{2}}+\frac{1}{R} \frac{\partial C}{\partial R}\right)-\operatorname{KrC}
\end{gathered}
$$

The corresponding initial and boundary conditions in dimensionless form are given as follows:

$$
\begin{aligned}
& \text { For all } X \text { and } R, \quad C=0, \quad T=0, \quad U=0, \quad V=0 \text {, } \\
& \text { at } R=1 \text {, } \\
& C=1, \quad T=1, \quad U=0, \quad V=0 \\
& \text { at } X=0 \\
& C=0, \quad T=0, \quad U=0, \quad V=0 \\
& \text { as } R \rightarrow \infty \\
& C \rightarrow 0, \quad T \rightarrow 0, \quad U \rightarrow 0, \quad V \rightarrow 0\} \\
& \left.\begin{array}{c}
\text { when } t \leq 0 \\
\text { when } t>0
\end{array}\right\}
\end{aligned}
$$

Similarly, the Eq. (9) also changed to the following dimensionless form.

$$
\frac{\partial U}{\partial R}=\frac{\partial V}{\partial X} \frac{1}{G r_{T}^{2}} \text { at } R=1 \text { and as } R \rightarrow \infty
$$




\subsection{Average momentum, heat transfer and mass transfer rates}

In view of real-life engineering applications, the researchers required the good knowledge on the values average momentum, heat and mass transfer rates. When compared to Nusselt and Sherwood numbers, skin friction coefficient pays the key role in the flow phenomena. In many of the technical applications, the raising skin-friction coefficient is unwanted but even though the raised skin-friction can be utilised in some applications such as thermal and mass exchanger's systems. In the current investigation, the momentum, thermal and concentration flow rates are obtained from the literature Rani et al. [16]. Thus, the dimensionless average momentum transport coefficient $\left(\bar{C}_{f}\right)$, heat $\operatorname{transfer}(\overline{N u})$ and mass transfer $(\overline{S h})$ rates are defined as follows:

$$
\begin{aligned}
& \bar{C}_{f}=\int_{0}^{1}\left(\frac{\partial U}{\partial R}\right)_{R=1} d X \\
& \overline{N u}=-\int_{0}^{1}\left(\frac{\partial T}{\partial R}\right)_{R=1} d X \\
& \overline{S h}=-\int_{0}^{1}\left(\frac{\partial C}{\partial R}\right)_{R=1} d X
\end{aligned}
$$

The differentials involved in Eqs. (17)-(19) are evaluated through a five-point formula, later integrals are computed through a Newton-Cotes scheme. Simulated dimensionless momentum, thermal and species diffusion rates for couple stress liquid are shown graphically and discussed below.

\section{Numerical solution procedure}

Governing non-dimensional unsteady fluid flow equations Eqs. (11)- (14) are simplified by employing implicit finite difference method. The associated finite difference equations are listed as follows:

$$
\begin{aligned}
& \frac{U_{l, m}^{n+1}-U_{l-1, m}^{n+1}+U_{l, m}^{n}-U_{l-1, m}^{n}}{2 \Delta X}+\frac{V_{l, m}^{n+1}-V_{l, m-1}^{n+1}+V_{l, m}^{n}-V_{l, m-1}^{n}}{2 \Delta R}+\frac{V_{l, m}^{n+1}}{(1+(m-1) \Delta R)}=0 \\
& \frac{U_{l, m}^{n+1}-U_{l, m}^{n}}{\Delta t}+\frac{U_{l, m}^{n}}{2 \Delta X}\left(U_{l, m}^{n+1}-U_{l-1, m}^{n+1}+U_{l, m}^{n}-U_{l-1, m}^{n}\right)+\frac{V_{l, m}^{n}}{4 \Delta R}\left(U_{l, m+1}^{n+1}-U_{l, m-1}^{n+1}+U_{l, m+1}^{n}-U_{l, m-1}^{n}\right) \\
& =\left(\frac{T_{l, m}^{n+1}+T_{l, m}^{n}}{2}\right)+B u\left(\frac{C_{l, m}^{n+1}+C_{l, m}^{n}}{2}\right)-\frac{1}{K 1}\left(\frac{U_{l, m}^{n+1}+U_{l, m}^{n}}{2}\right)-M\left(\frac{U_{l, m}^{n+1}+U_{l, m}^{n}}{2}\right) \\
& +\left(\frac{\left.U_{l, m+1}^{n+1}-2 U_{l, m}^{n+1}+U_{l, m-1}^{n+1}+U_{l, m+1}^{n}-2 U_{l, m}^{n}+U_{l, m-1}^{n}\right)+\left(\frac{U_{l, m+1}^{n+1}-2 U_{l, m}^{n+1}+U_{l, m-1}^{n+1}+U_{l, m+1}^{n}-2 U_{l, m}^{n}+U_{l, m-1}^{n}}{2 \Delta R^{2}}\right)}{2(1+(m-1) \Delta R)^{2} \Delta R^{2}}\right) \\
& +\left(\frac{U_{l, m+1}^{n+1}-U_{l, m-1}^{n+1}+U_{l, m+1}^{n}-U_{l, m-1}^{n}}{4(1+(m-1) \Delta R) \Delta R}\right)-\left(\frac{U_{l, m+1}^{n+1}-U_{l, m-1}^{n+1}+U_{l, m+1}^{n}-U_{l, m-1}^{n}}{4(1+(m-1) \Delta R)^{3} \Delta R}\right) \\
& -\left(\frac{U_{l, m+2}^{n+1}-2 U_{l, m+1}^{n+1}+2 U_{l, m-1}^{n+1}-U_{l, m-2}^{n+1}+U_{l, m+2}^{n}-2 U_{l, m+1}^{n}+2 U_{l, m-1}^{n}-U_{l, m-2}^{n}}{2(1+(m-1) \Delta R) \Delta R^{3}}\right) \\
& -\left(\frac{U_{l, m+2}^{n+1}-4 U_{l, m+1}^{n+1}+6 U_{l, m}^{n+1}-4 U_{l, m-1}^{n+1}+U_{l, m-2}^{n+1}+U_{l, m+2}^{n}-4 U_{l, m+1}^{n}+6 U_{l, m}^{n}-4 U_{l, m-1}^{n}+U_{l, m-2}^{n}}{2 \Delta R^{4}}\right)
\end{aligned}
$$




$$
\begin{aligned}
& \frac{T_{l, m}^{n+1}-T_{l, m}^{n}}{\Delta t}+\frac{U_{l, m}^{n}}{2 \Delta X}\left(T_{l, m}^{n+1}-T_{l-1, m}^{n+1}+T_{l, m}^{n}-T_{l-1, m}^{n}\right)+\frac{V_{l, m}^{n}}{4 \Delta R}\left(T_{l, m+1}^{n+1}-T_{l, m-1}^{n+1}+T_{l, m+1}^{n}-T_{l, m-1}^{n}\right) \\
= & \frac{1}{P r}\left(1+\frac{4}{3 N r}\right)\left(\frac{T_{l, m+1}^{n+1}-2 T_{l, m}^{n+1}+T_{l, m-1}^{n+1}+T_{l, m+1}^{n}-2 T_{l, m}^{n}+T_{l, m-1}^{n}}{2 \Delta R^{2}}\right) \\
& +\frac{1}{\operatorname{Pr}}\left(1+\frac{4}{3 N r}\right)\left(\frac{T_{l, m+1}^{n+1}-T_{l, m-1}^{n+1}+T_{l, m+1}^{n}-T_{l, m-1}^{n}}{4(1+(m-1) \Delta R) \Delta R}\right)+E c\left(\frac{U_{l, m}^{n+1}-U_{l, m-1}^{n+1}+U_{l, m+1}^{n}-U_{l, m-1}^{n}}{4 \Delta R}\right)^{2} \\
& \frac{C_{l, m}^{n+1}-C_{l, m}^{n}}{\Delta t}+\frac{U_{l, m}^{n}}{2 \Delta X}\left(C_{l, m}^{n+1}-C_{l-1, m}^{n+1}+C_{l, m}^{n}-C_{l-1, m}^{n}\right)+\frac{V_{l, m}^{n}}{4 \Delta R}\left(C_{l, m+1}^{n+1}-C_{l, m-1}^{n+1}+C_{l, m+1}^{n}-C_{l, m-1}^{n}\right) \\
= & \frac{1}{S c}\left(\frac{C_{l, m+1}^{n+1}-C_{l, m-1}^{n+1}+C_{l, m+1}^{n}-C_{l, m-1}^{n}}{4(1+(m-1) \Delta R) \Delta R}\right)+\frac{1}{S c}\left(\frac{C_{l, m+1}^{n+1}-2 C_{l, m}^{n+1}+C_{l, m-1}^{n+1}+C_{l, m+1}^{n}-2 C_{l, m}^{n}+C_{l, m-1}^{n}}{2 \Delta R^{2}}\right) \\
- & \operatorname{Kr}\left(\frac{C_{l, m}^{n+1}+C_{l, m}^{n}}{2}\right)
\end{aligned}
$$

The solutions of the Eqs. (20)-(23) are obtained in the rectangular region of the geometry limiting from $X_{\min }=0$, $X_{\max }=1, R_{\min }=1$ and $R_{\max }=20$, here $R_{\max }$ assuming as $R=\infty$ be located far from the momentum, heat and mass diffusion boundary layers. Unsteady velocity, heat and mass diffusion values are obtained using the mesh space of 100 $\times 500$ varies $2^{\text {nd }}$ decimal with $50 \times 250$ and $50 \times 250$ differing in $5^{\text {th }}$ decimal with $200 \times 100$. The mesh space of $100 \times$ 500 has been finalised for all numerical computations with 0.03 and 0.01 as mesh sizes along $R$ and $X$ directions. Also, the step size of time dependence has verified with $\Delta t=0.01$ and provides good result. Further, for unsteady solutions of velocity, temperature and concentration, the absolute difference between the flow field at two successive times is smaller than $10^{-5}$ at all mesh nodes is maintained.

By solving the energy and concentration Eqs. (22)-(23), the temperature and mass diffusion fields are obtained, respectively. Further, the result of momentum and continuity equations, gives the velocity field. Finite difference equations of Eqs. (21)-(23) at $(n+1)^{\text {th }}$ computation can be written in pentdiagonal and tridiagonal formulae, respectively as listed below.

$$
\begin{gathered}
A_{1} \Psi_{l, m-2}^{n+1}+B_{1} \Psi_{l, m-1}^{n+1}+C_{1} \Psi_{l, m}^{n+1}+D_{1} \Psi_{l, m+1}^{n+1}+E_{1} \Psi_{l, m+2}^{n+1}=F_{1} \\
A_{2} \varphi_{l, m-1}^{n+1}+B_{2} \varphi_{l, m}^{n+1}+C_{2} \varphi_{l, m+1}^{n+1}=D_{2}
\end{gathered}
$$

where $\Psi$ represents the dependent variable velocity $U$ and $\varphi$ represents the dependent variables $T$ and $C$. Therefore, the Eqs. (24) and (25) at each interior node on a specific $l$-level creates a pentadiagonal and tridiagonal equations. These equations are simplified through pentadiagonal and Thomas schemes. Explicitly the velocity $V$ is found from the finite difference equation Eq. (20). This mechanism is continual for all successive $l$-levels with more time steps till the convergence criteria is attained $10^{-5}$.

\section{Results and discussions}

Based on the present numerical analysis following results are demonstrated. To study the time-dependent behaviour of simulated patterns such as flow field, thermal, concentration and their numerical values at various places are shown and which are neighbouring to heated surface of vertical cylinder. At $X=1.0$ along $R$-coordinate, the steady mass diffusion, thermal and flow fields are described.

\subsection{Validation of currents with existing solutions}

The steady-state simulated mass diffusion fields of couple stress liquid obtained in the current investigation is compared with the existing results of Rani et al. [17]. It is clear from Figure 2 that, the current findings are showing accurate matching with the existing solutions. 


\subsection{Analysis of numerical results}

Current numerical solutions are expressed in terms of magnetic parameter $(M)$, porous medium parameter $(K 1)$, radiation number $(\mathrm{Nr})$, chemical reaction number $(\mathrm{Kr})$ Buoyancy ratio parameter $(\mathrm{Bu})$ and Eckert number and rest of the variables kept constant.

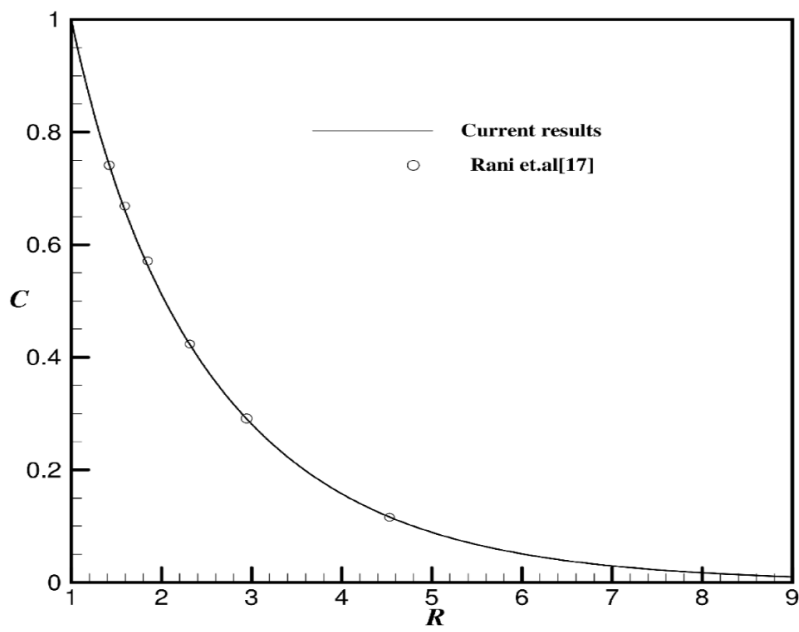

Figure 2: Validation of current results with the existed results of Rani et al. [17].

\section{(i) Effect of magnetic field $(M)$ on flow profiles}

Velocity profile: The simulated velocity field $U$ at position $(1,1.38)$ verses $t$ is illustrated in Figure 3(a). It is determined that, the unsteady-state flow field decayed with rising $M$. It is because of fact that, the increasing magnetic number amplifies the Lorenz forces in the flow region and hence velocity profile is diminished. Also, the boundary layer thickness diminished with rising $M$. It is witnessed that, for the enhancing $M$, the maximum velocity peaks diminished. Also, the time required to reach the time-independent state increases with amplifying $M$. Initially (i.e., $t \ll 1$ ) velocity profiles are coinciding with each other due to convective thermal transfer process is dominated by the conduction heat transfer.

The computer generated flow field $U$ verses $R$ at $X=$ 1 is demonstrated in the Figure $3(\mathrm{~b})$ under the action of magnetic field. Initially flow field begun with zero near the cylindrical hot surface, touch its greatest values and monotonically decays to value zero along $R$-direction for all $t$. It is recorded from Figure 3(b) that, the steady-state velocity acts as a decaying function of magnetic parameter.

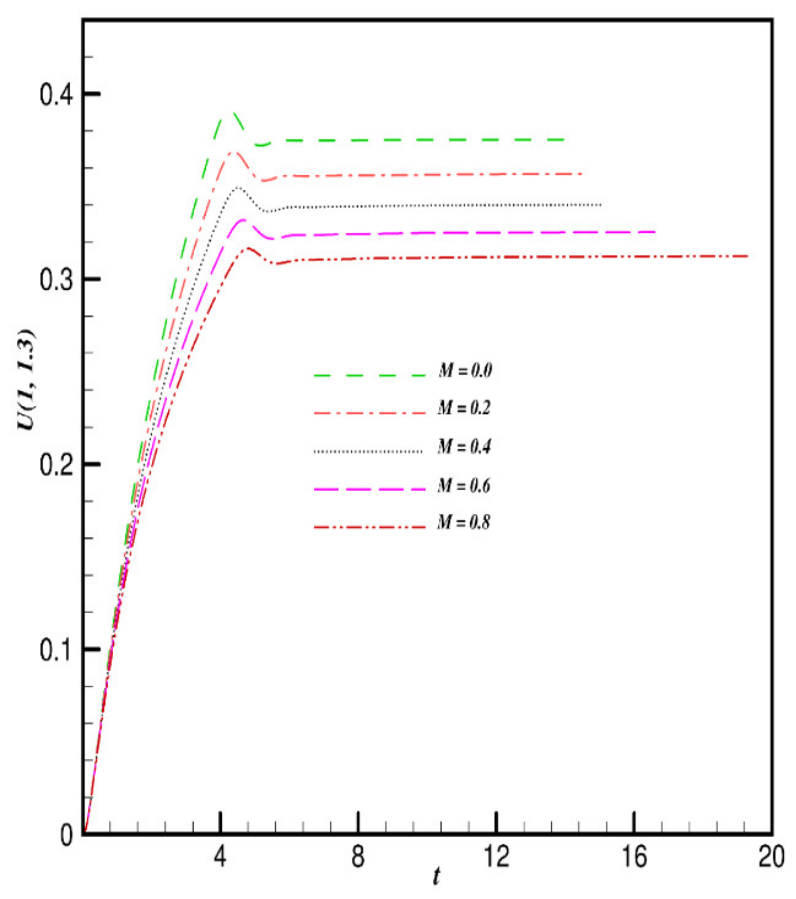

(a)

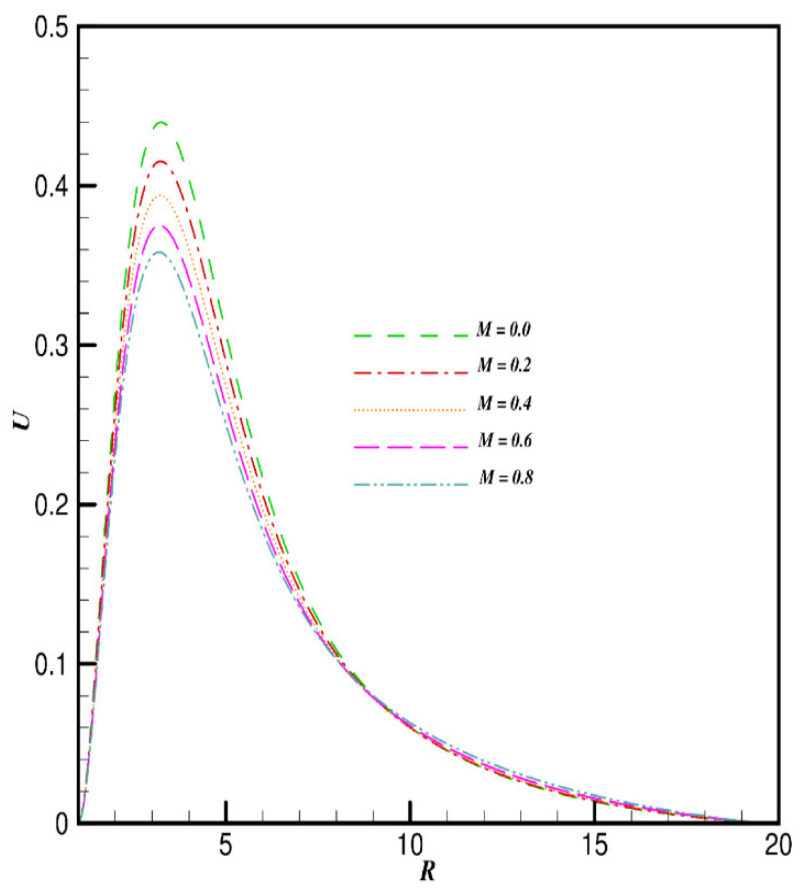

(b)

Figure 3: $(a)$ Transient and $(b)$ Steady state velocity profiles with fixed $S c=0.6, P r=0.7, B u=1.0, K r=-0.2, N r=2.0, K 1=1.0$, $E c=0.1$. 


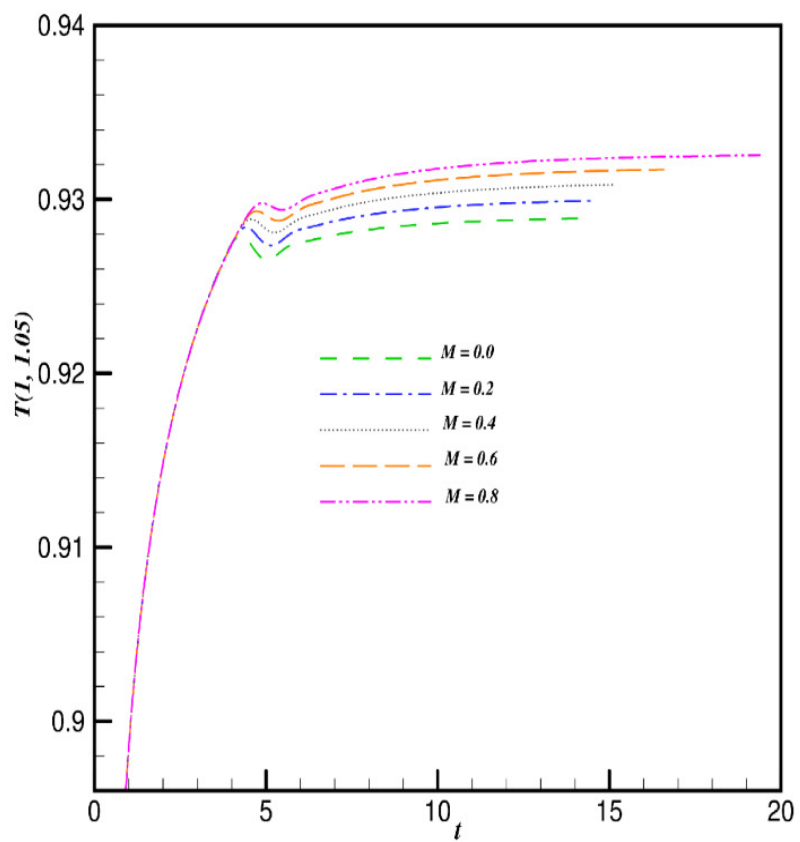

(a)

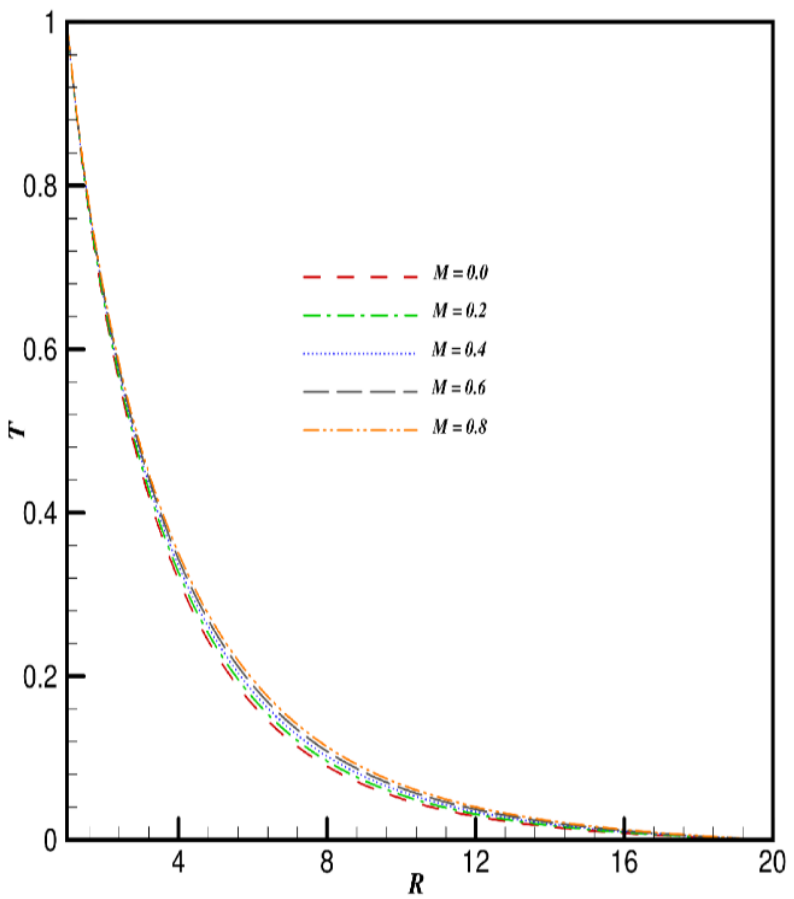

(b)

Figure 4: $(a)$ Transient and $(b)$ Steady state temperature profiles with fixed $S c=0.6, \mathrm{Pr}=0.7, \mathrm{Bu}=1.0, \mathrm{Kr}=-0.2, \mathrm{Nr}=2.0$, $K 1=1.0, E c=0.1$.

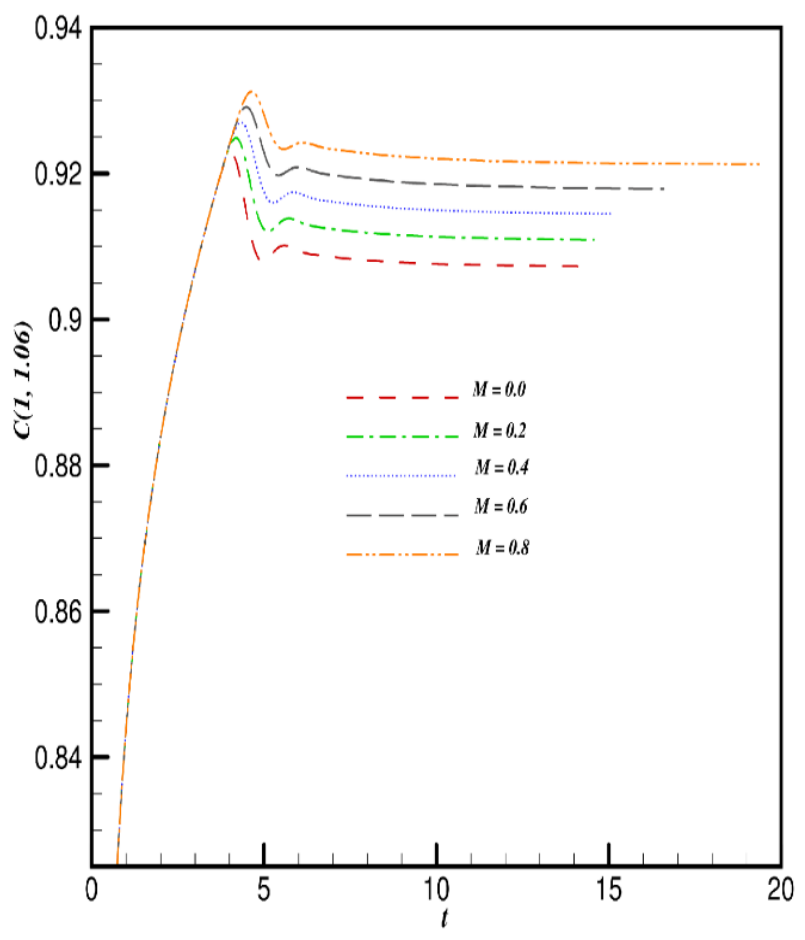

(a)

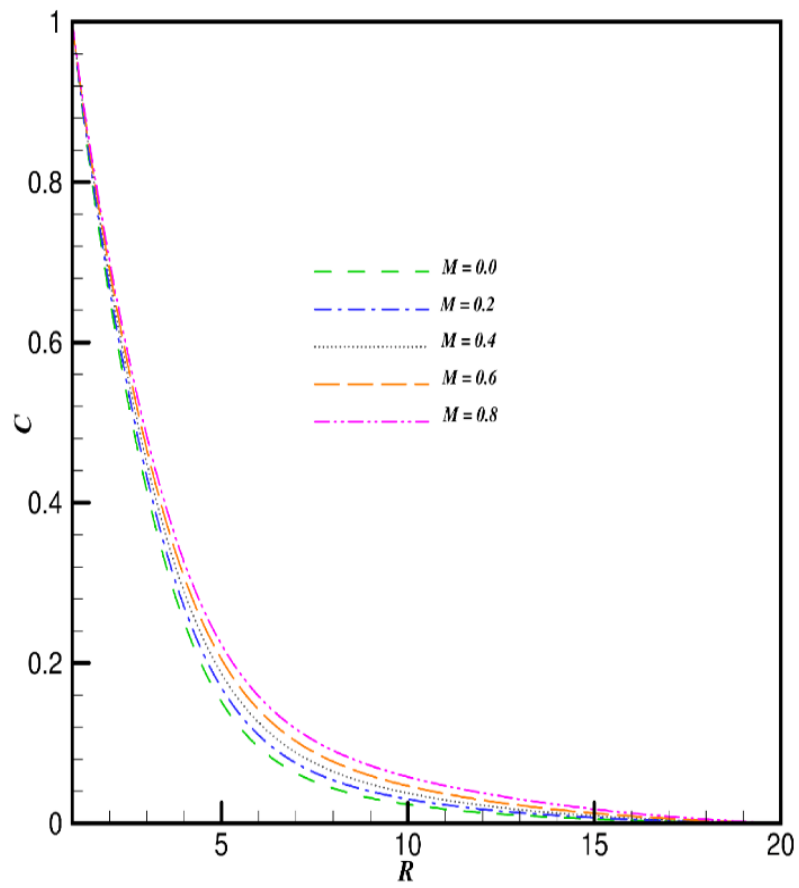

(b)

Figure 5: $(a)$ Transient and (b) Steady state concentration profiles with fixed $S c=0.6, P r=0.7, B u=1.0, K r=-0.2, N r=2.0$, $K 1=1.0, E c=0.1$. 

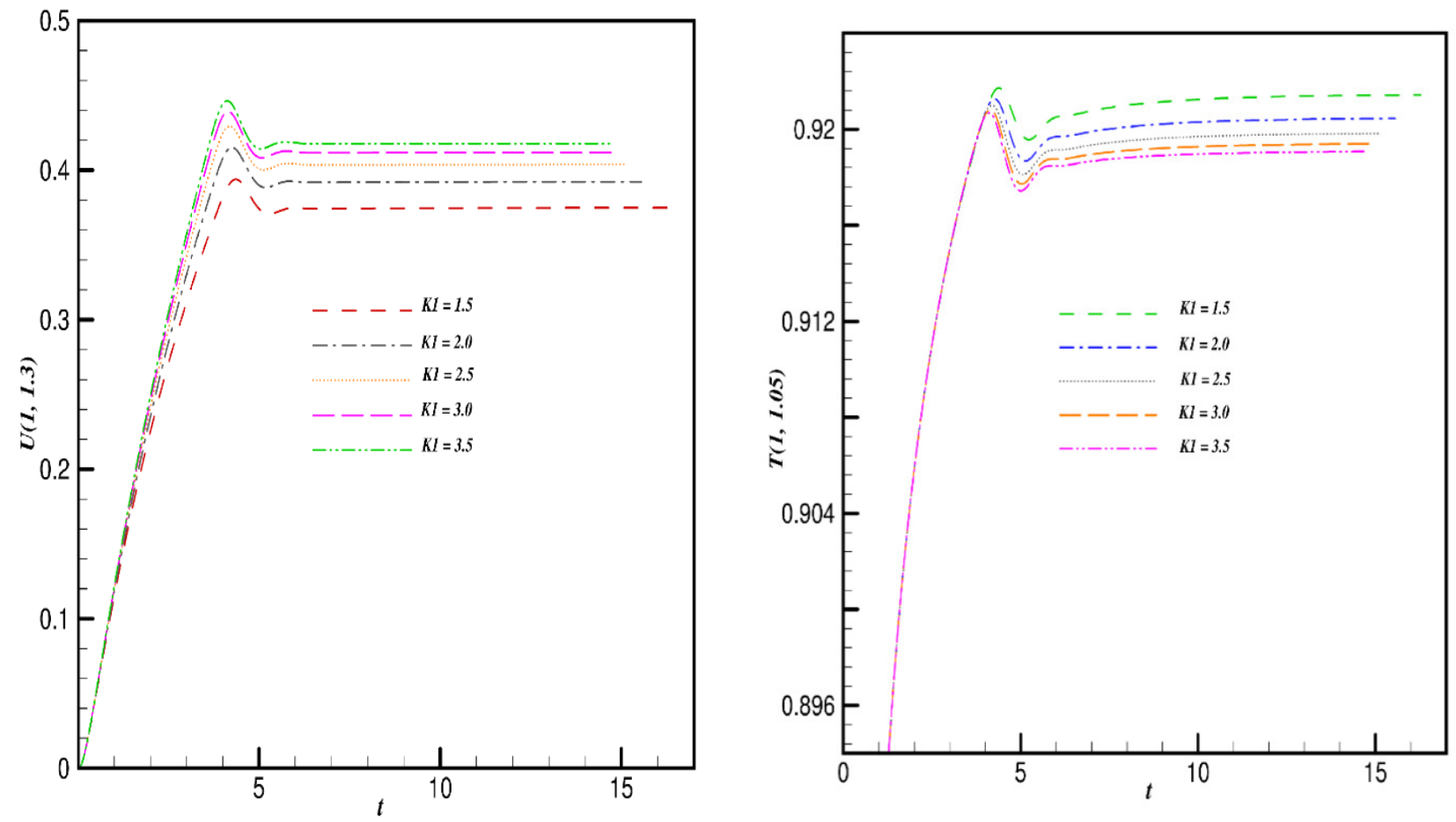

(a)

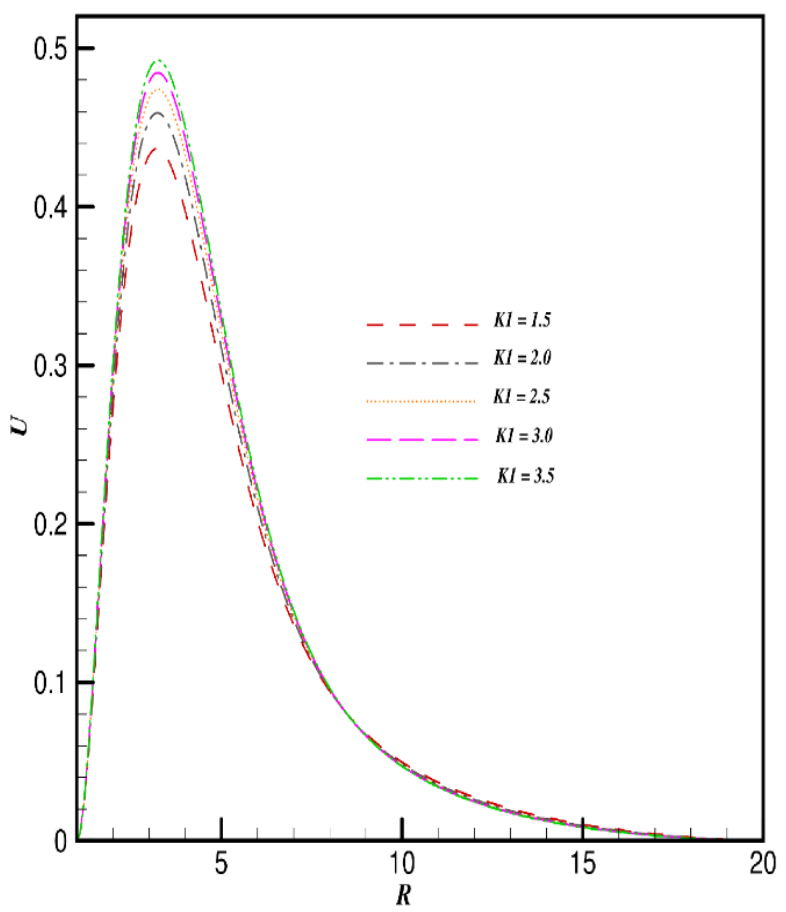

(b)

Figure 6: $(a)$ Transient and (b) Steady state velocity profiles with fixed $S c=0.6, P r=0.7, B u=1.0, K r=-0.2, N r=1.0, M=0.2$, $E c=0.1$. (a)

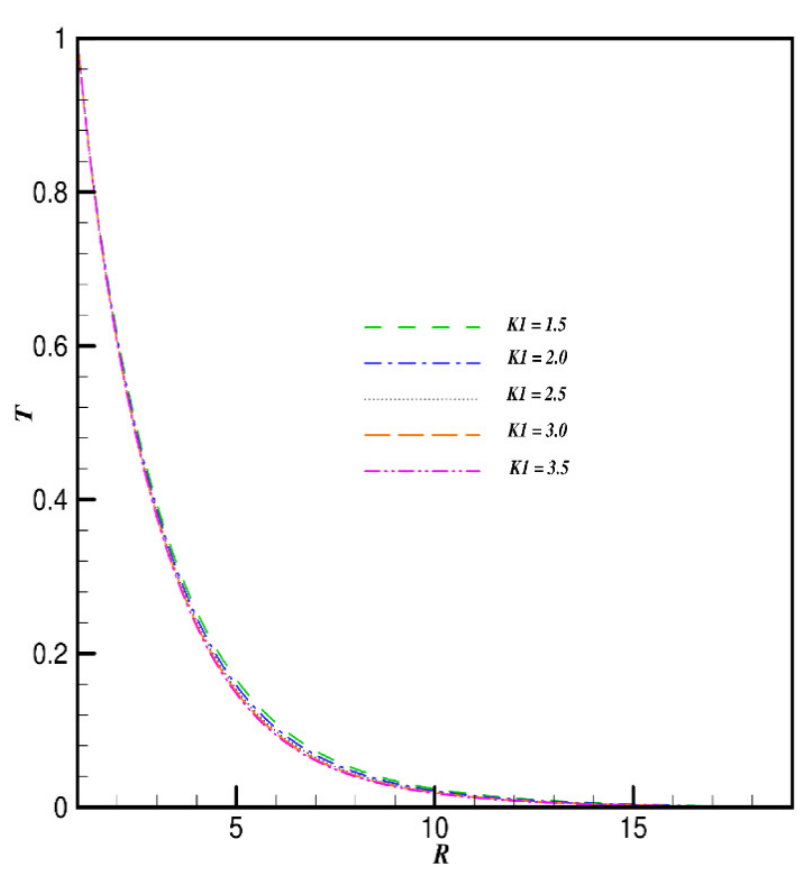

a)

(b)

Figure 7: $(a)$ Transient and $(b)$ Steady state temperature profiles with fixed $S c=0.6, P r=0.7, B u=1.0, K r=-0.2, N r=1.0$, $M=0.2, E c=0.1$. 

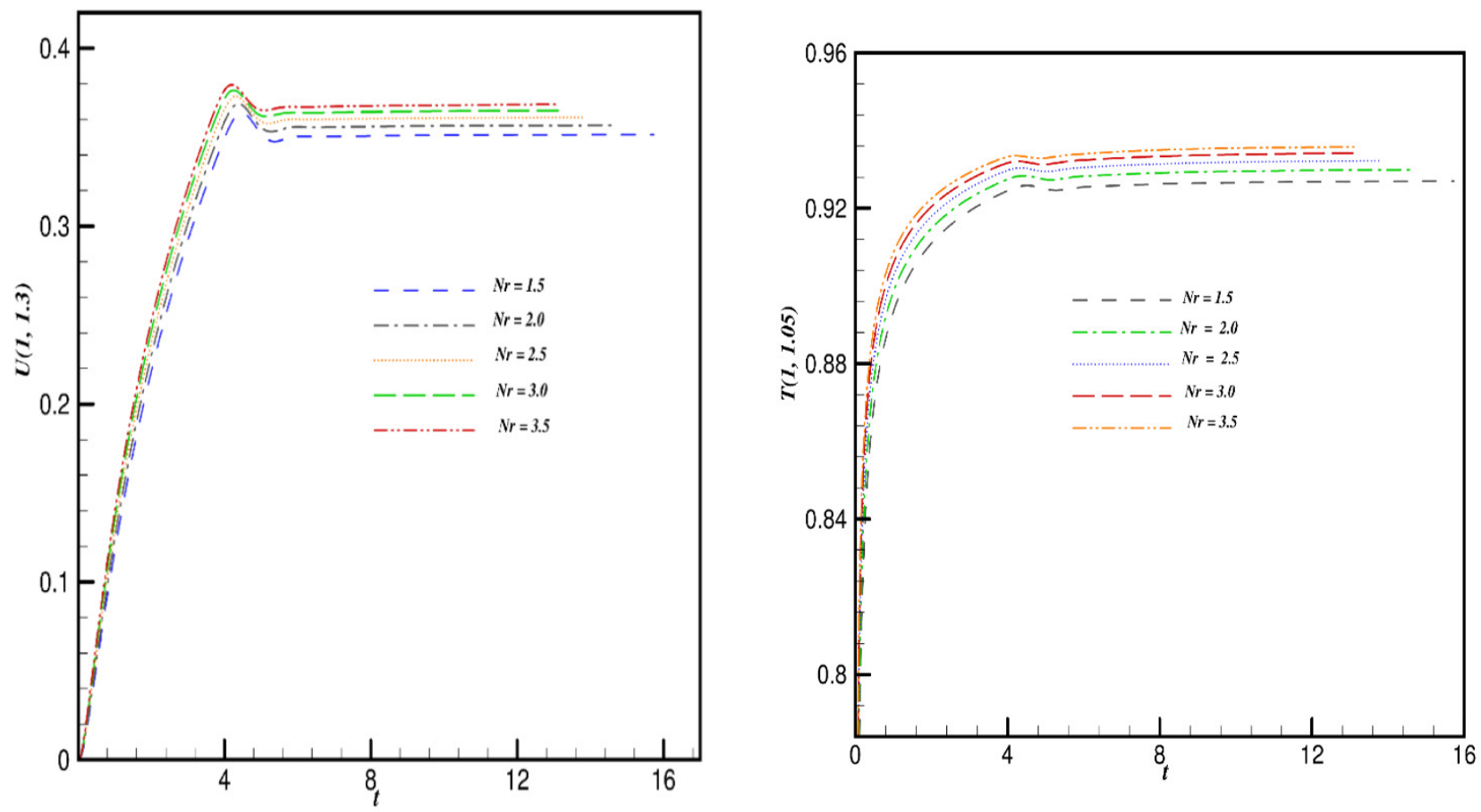

(a)

(a)
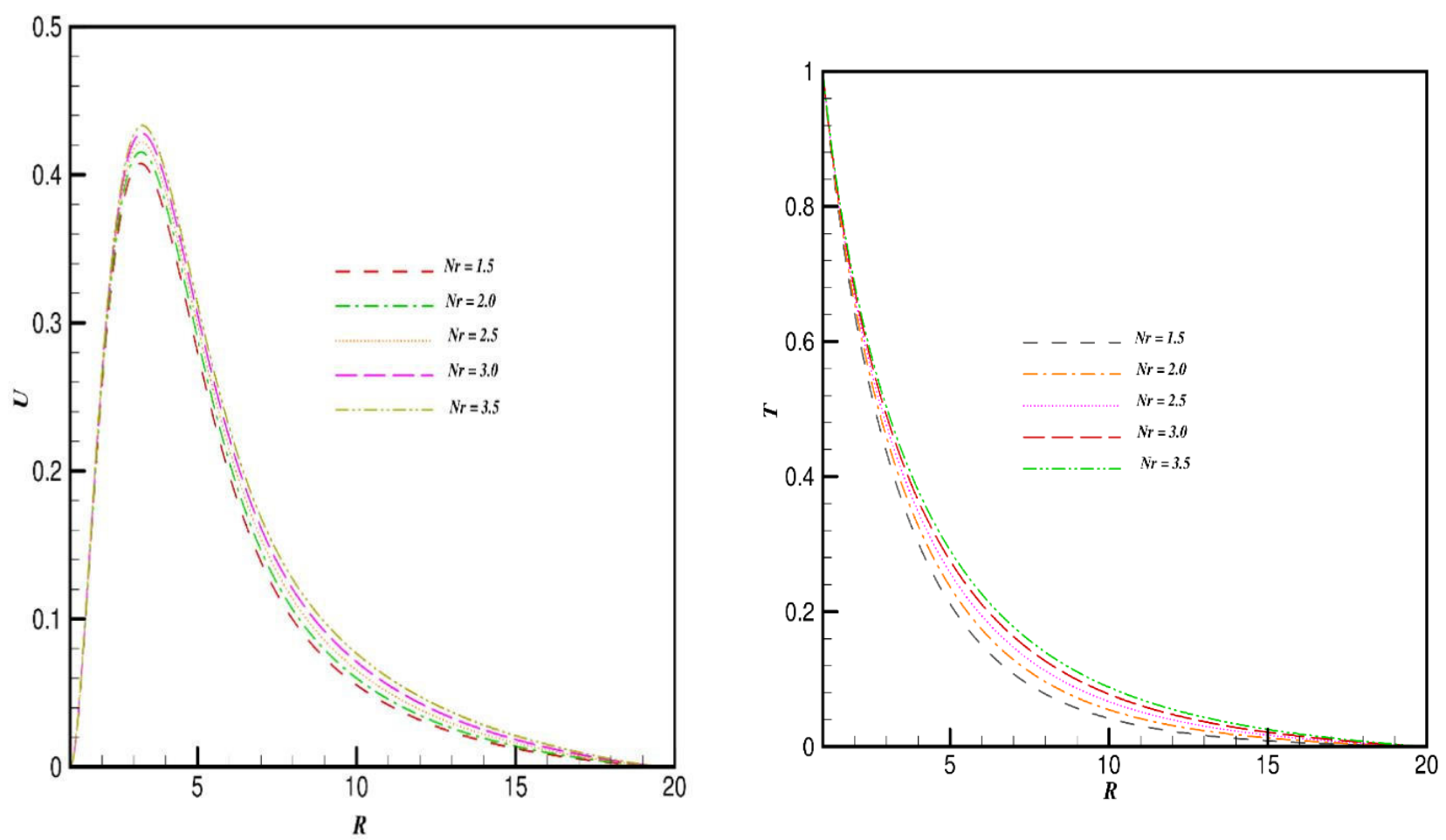

(b)

(b)

Figure 8: $(a)$ Transient and $(b)$ Steady state velocity profiles with fixed $S c=0.6, P r=0.7, B u=1.0, K r=-0.2, K 1=1.0, M=0.2$, $E c=0.1$.

Figure 9: $(a)$ Transient and (b) Steady state temperature profiles with fixed $S c=0.6, P r=0.7, B u=1.0, K r=-0.2, K 1=1.0$, $M=0.2, E c=0.1$. 


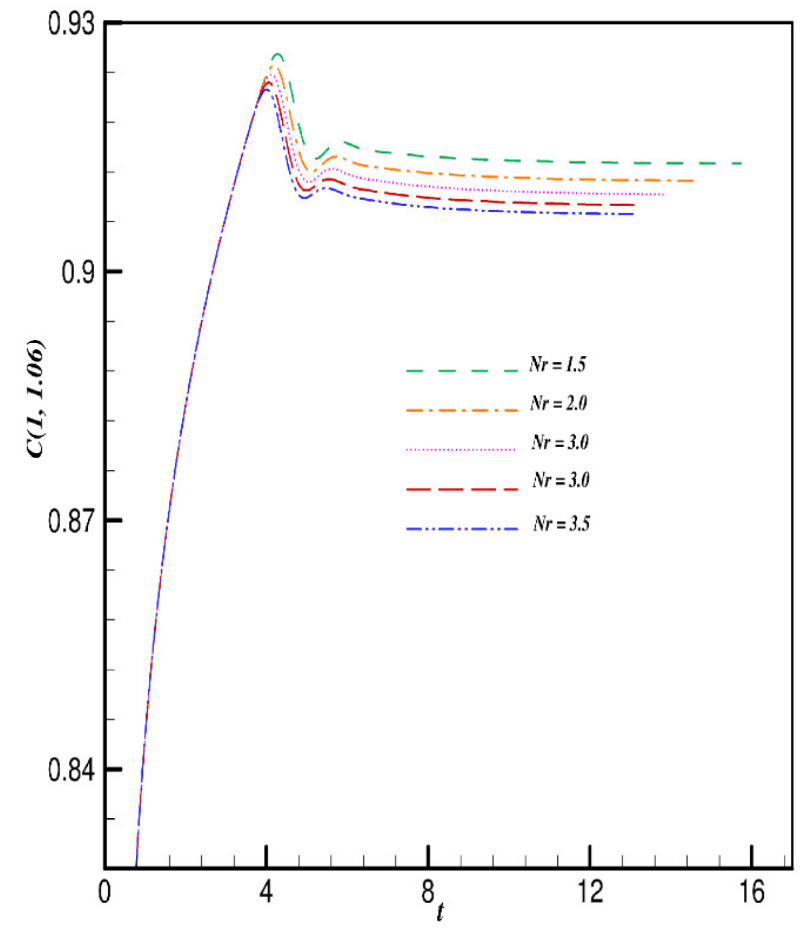

(a)

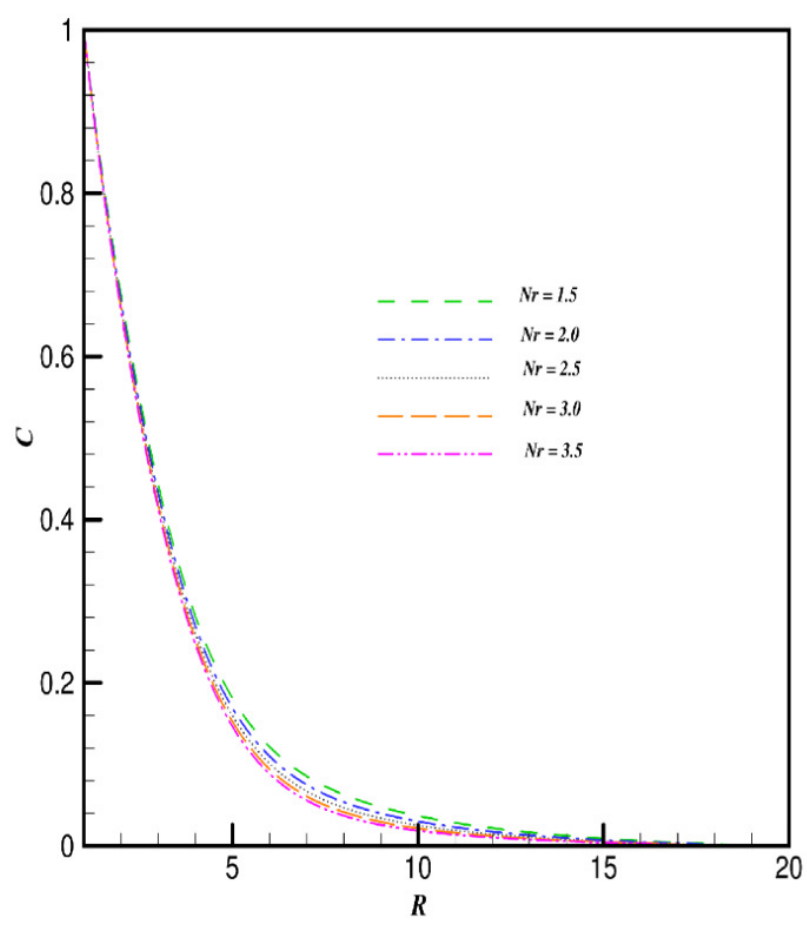

(b)

Figure 10: $(a)$ Transient and $(b)$ Steady state concentration profiles with fixed $S c=0.6, P r=0.7, B u=1.0, K r=-0.2, K 1=1.0$, $M=0.2, E c=0.1$.

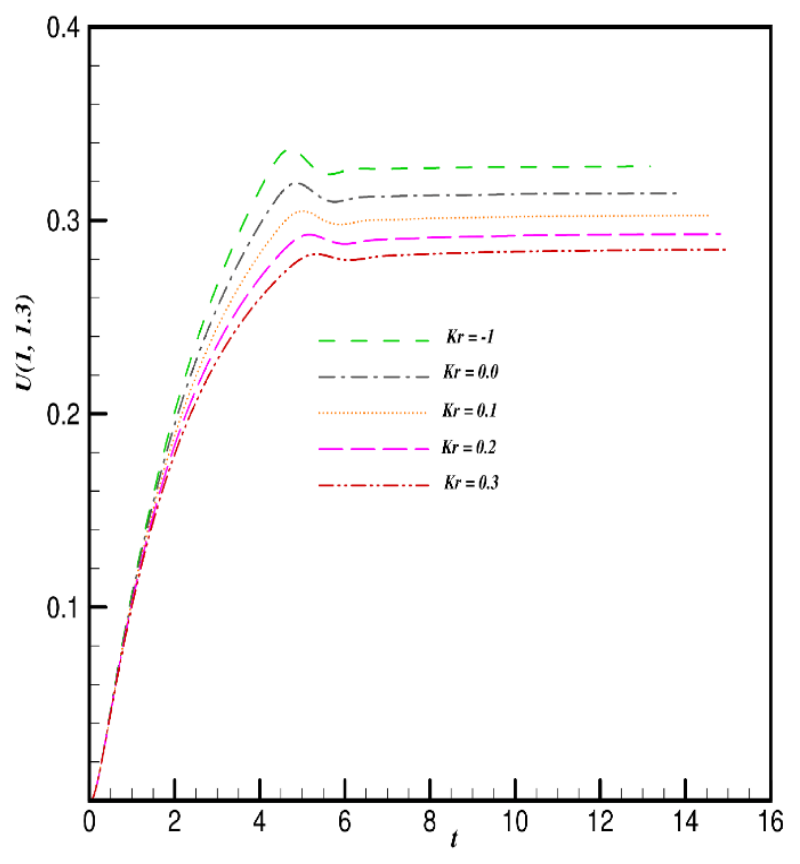

(a)

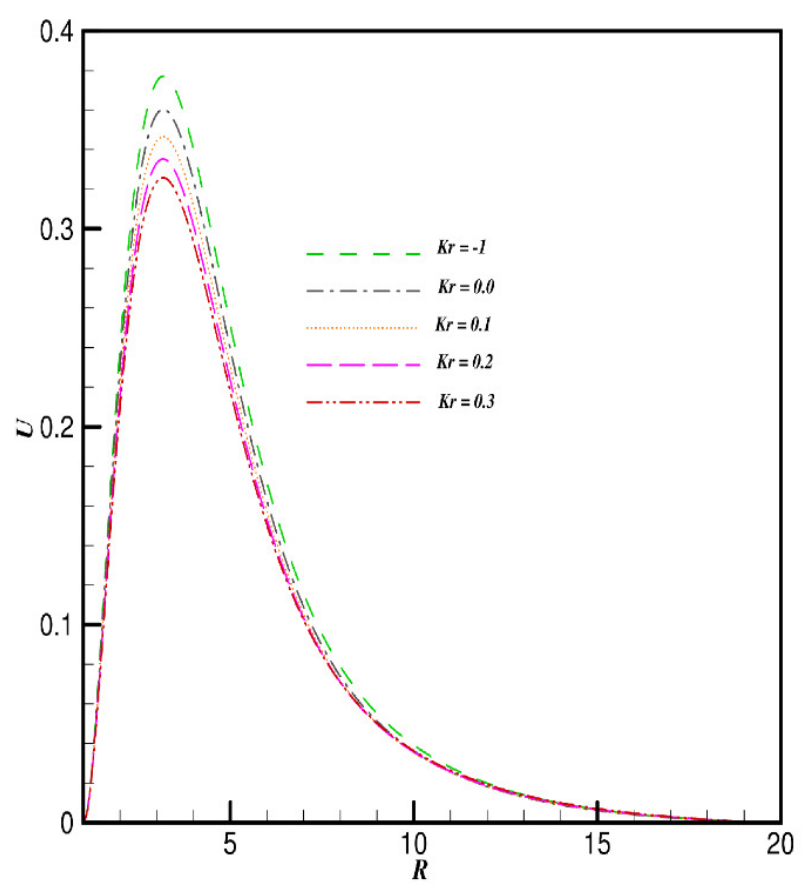

(b)

Figure 11: $(a)$ Transient and (b) Steady state velocity profiles with fixed $S c=0.6, P r=0.7, B u=1.0, N r=1.0, K 1=1.0, M=0.2$, $E c=0.1$. 


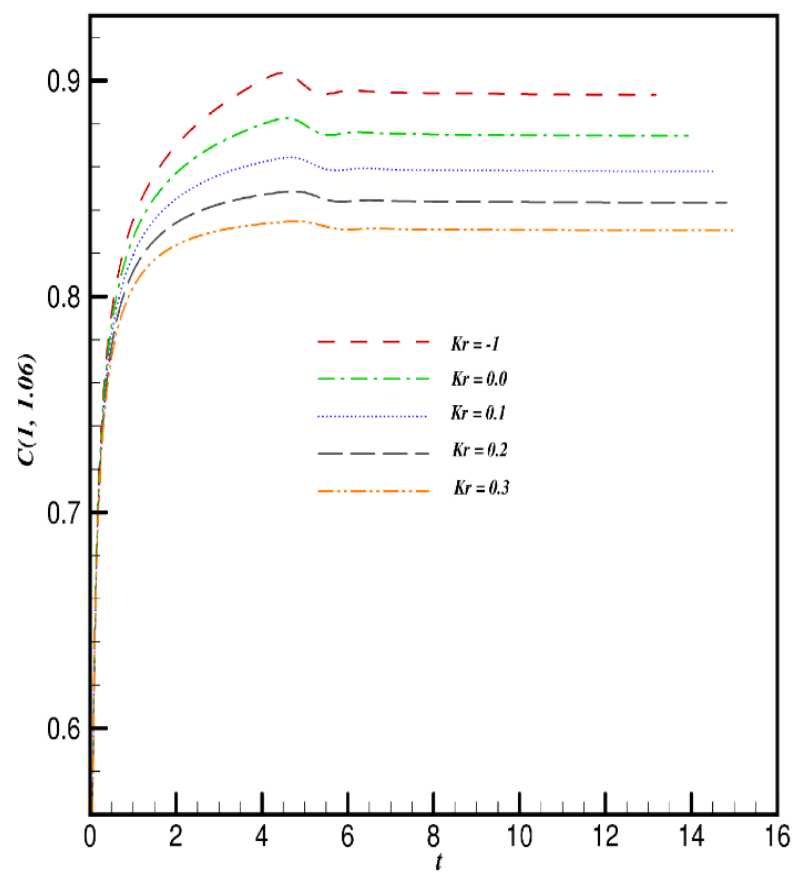

(a)

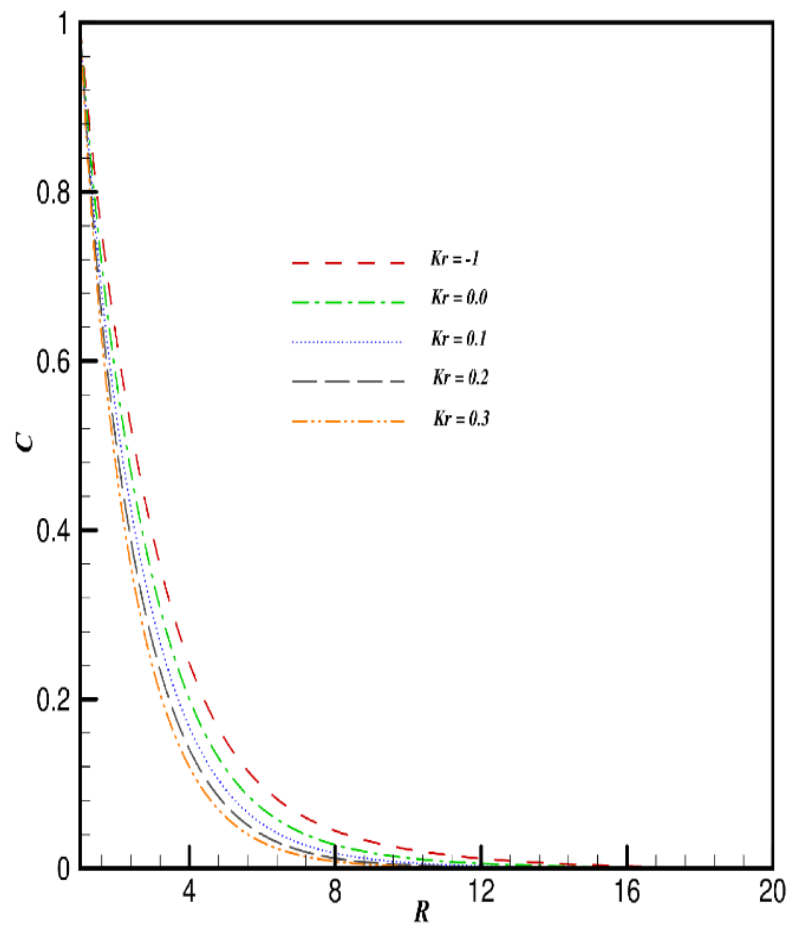

(b)

Figure 12: $(a)$ Transient and $(b)$ Steady state concentration profiles with fixed $S c=0.6, P r=0.7, B u=1.0, N r=1.0, K 1=1.0$, $M=0.2, E c=0.1$.

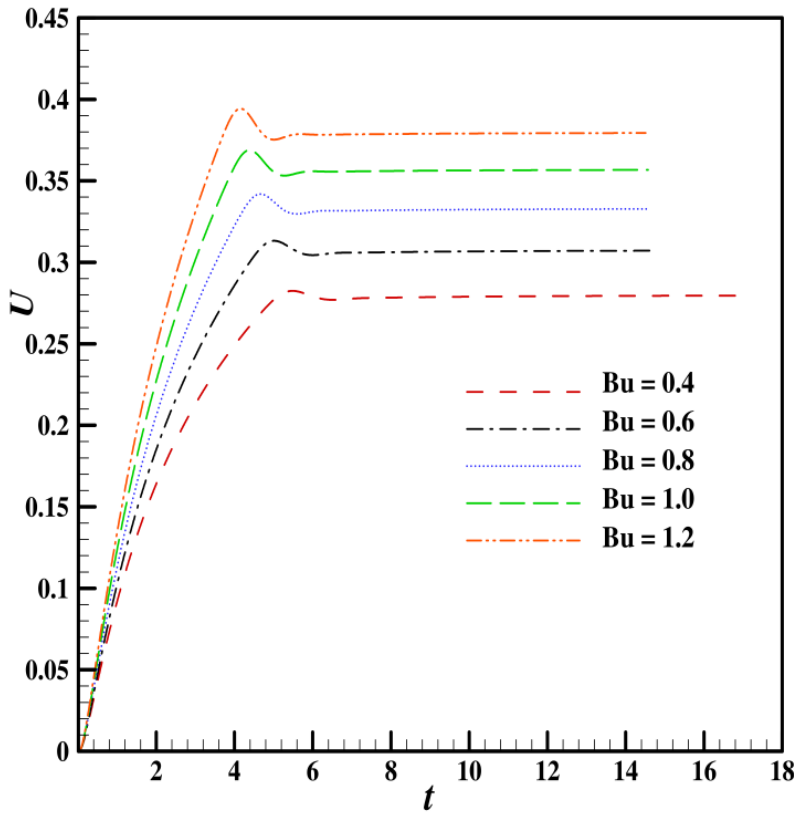

(a)

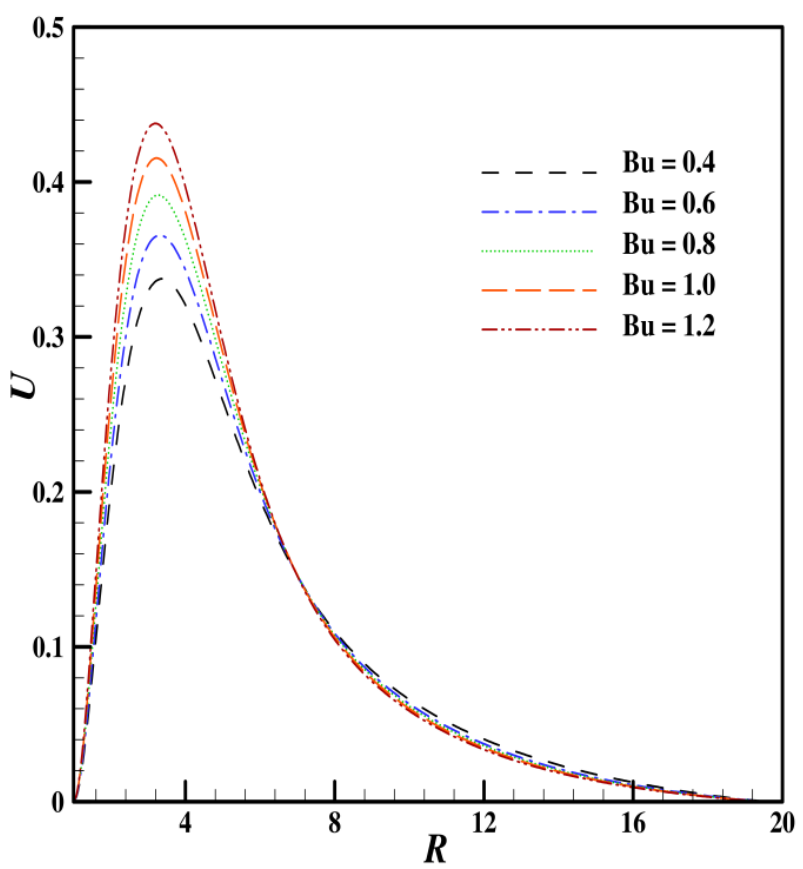

(b)

Figure 13: $(a)$ Transient and $(b)$ Steady state velocity profiles with fixed $S c=0.6, P r=0.7, K r=-0.2, N r=1.0, K 1=1.0, M=0.2$, $E c=0.1$. 


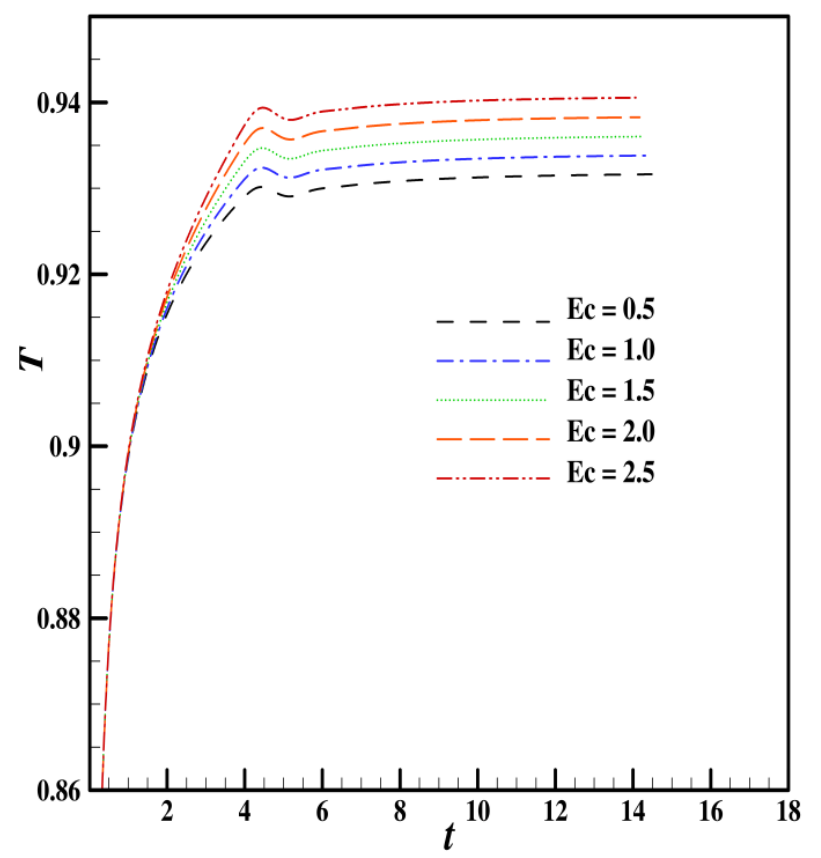

(a)

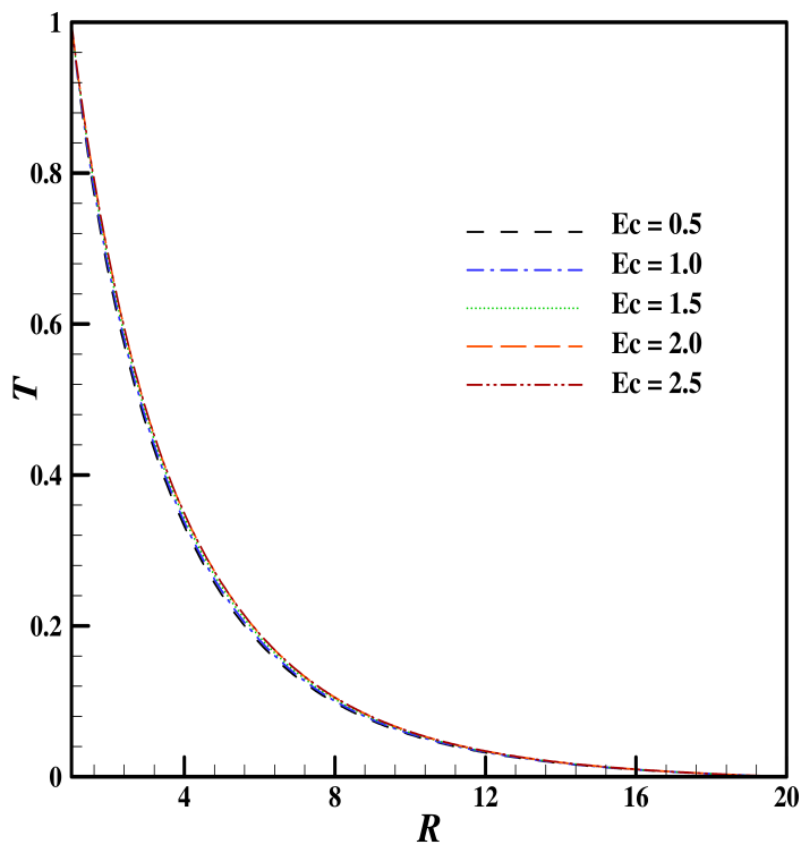

(b)

Figure 14: $(a)$ Transient and $(b)$ Steady state temperature profiles with fixed $S c=0.6, \mathrm{Pr}=0.7, \mathrm{Kr}=-0.2, \mathrm{Bu}=1.0, \mathrm{Nr}=1.0$, $K 1=1.0, M=0.2$.
Temperature profile: The simulated transient temperature profile with enhancing values of magnetic field $M$ at $(1,1.05)$ is demonstrated in Figure 4(a). This plot shows that, the merged thermal curves indicates that, initially conduction process dominates the convection phenomena. Transient temperature profile rises with time, reaches maximum values, shortly later, diminished and again gradually increases, at last asymptotically reached steady state. Unsteady temperature profile rises with amplifying values of magnetic parameter.

At $X=1.0$ along radial axis, the time-independent temperature profile is shown in the Figure 4(b) for the giving different values of $M$. The temperature curves originate from the hot cylinder wall at $T=1$ and diminished monotonically to zero irrespective of time along the radial coordinate. However, steady state velocity profile enhanced with increasing $M$.

Concentration profile: The computer-generated unsteady concentration diffusion field for the various magnetic parameter $M$ values at location $(1,1.06)$ is displayed in the Figure 5(a). Initially all the concentration diffusion arcs are fully merged with one another and diverged after some time. Also, it is noticed that, the unsteady concentration field increases, attains maximum as well as minimum and again increases, there after decreases, later asymptotically attains the stable state condition. Further, for the rising $M$, the mass diffusion field increases.

The time-independent simulated concentration profile at $X=1.0$ verses $R$ for various values of $M$ is shown in the Figure 5(b). The concentration diffusion curves are originated from the heated cylinder wall at $C=1$ and diminished monotonically to zero along $R$-axis irrespective of $t$. Steady-state concentration profile rises with rising $M$ values.

(ii) Effect of porous parameter (K1) on velocity and temperature profile

Velocity Profile: The simulated time-dependent velocity field for different values of porous parameter $K 1$ is shown in the Figure 6(a) at the location $(1,1.3)$. It is found from this figure that, the enhancing porous parameter amplifies the velocity profiles. According to Darcy's law, the flow between the two points is directly related to the distance between points, pressure difference between the points and connectivity of the flow between the points within the medium. Thus, an increment in porous number results the free flow of couple stress fluid in the medium, hence velocity boundary layer thickness increases. It is shown that velocity profile slowly rises with time, attained maximum around $t=4.5$ and then decreases attain the temporal minima and again increases, decreases slightly, later attain the steady-state. 

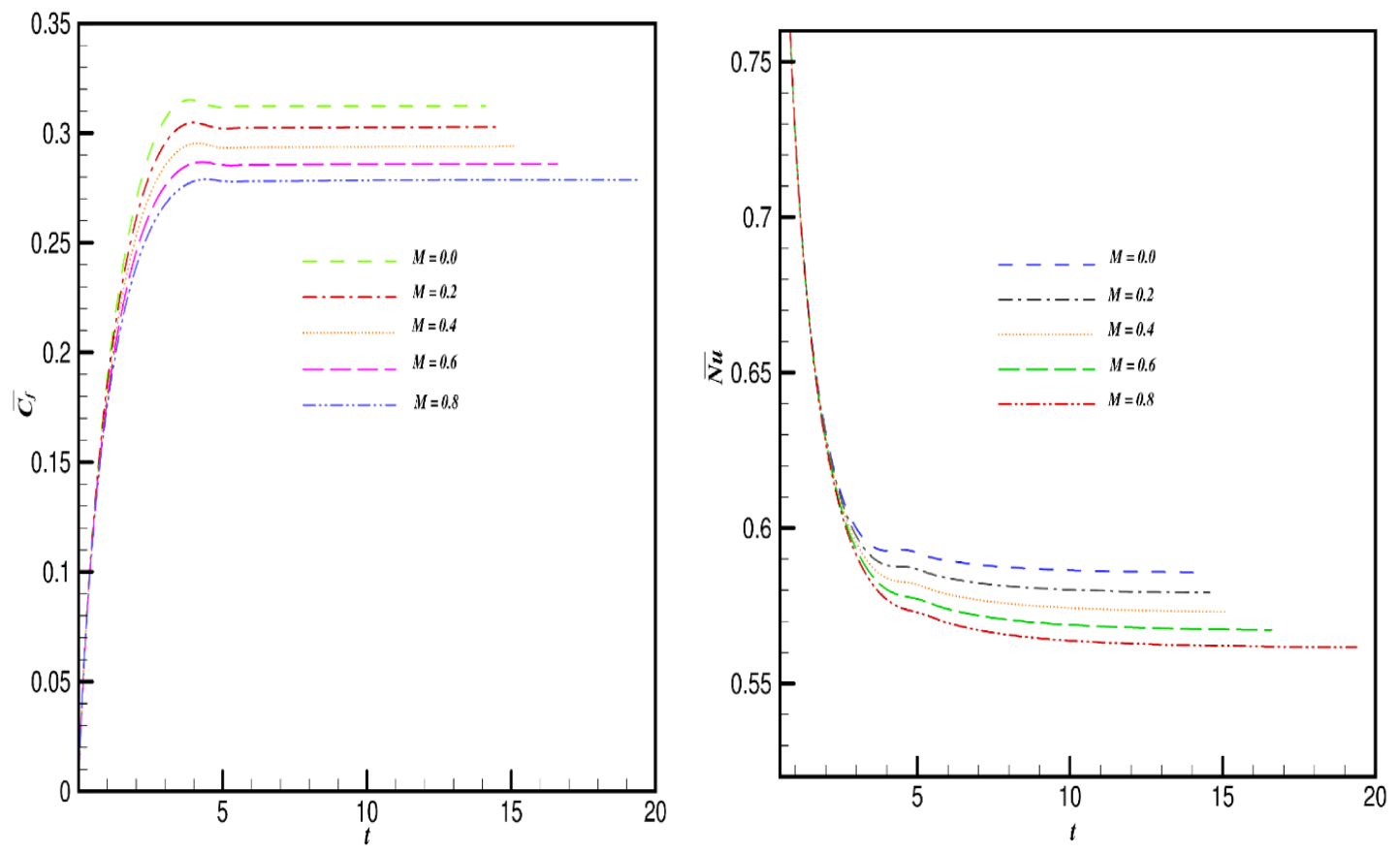

(a)

(b)

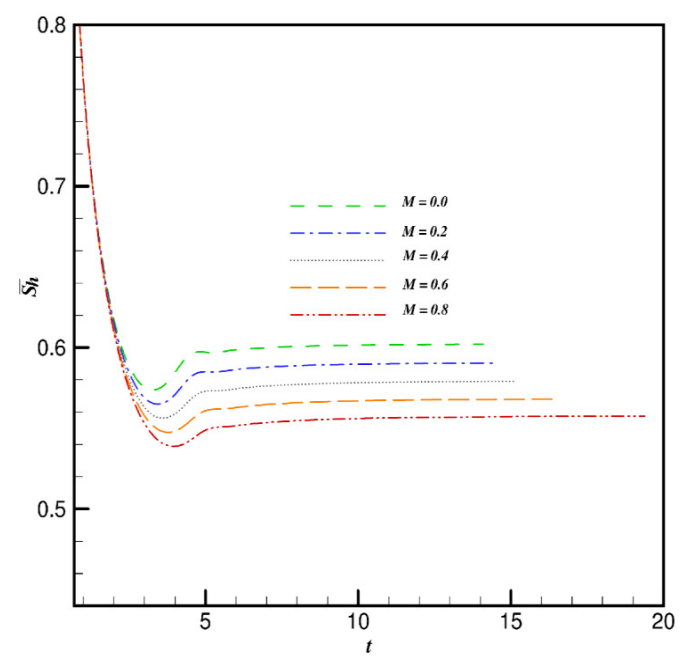

(c)

Figure 15: Profiles of average (a) skin friction coefficient, $(b)$ Nusselt numer and (c) Sherwood number for fixed $S c=0.6, P r=0.7, B u=$ 1.0, $K r=-0.2, N r=2.0, K 1=1.0, E c=0.1$.

The time-independent simulated velocity profile at $X=1$ verses $R$ is demonstrated in the Figure 6(b) for various $K 1$ values. This graph shows that, the flow field starts initially zero at heated wall of cylinder, reaches the highest value and shortly later diminished to zero monotonically in $R$-direction. Also, for the increasing values of $K 1$, the steady state velocity profile are increases.

Temperature profile: The Figure $7(\mathrm{a})$ at $(1,1.05)$ shows the simulated unsteady temperature profile for var- ious $K 1$ values. Temperature profile diminished with rising $K 1$. It is due to fact that, the rising $K 1$ raises the flow between the points and pressure difference between the points decreases, then this decrease in the heat generation. Also, noticed that, initially all the thermal curves merged with each other, shortly later reaches temporal maxima as well as minima and again increases, later it attains the steady state. The Figure $7(\mathrm{~b})$ shows the simulated steady state temperature profile at $X=1.0$ against $R$ 


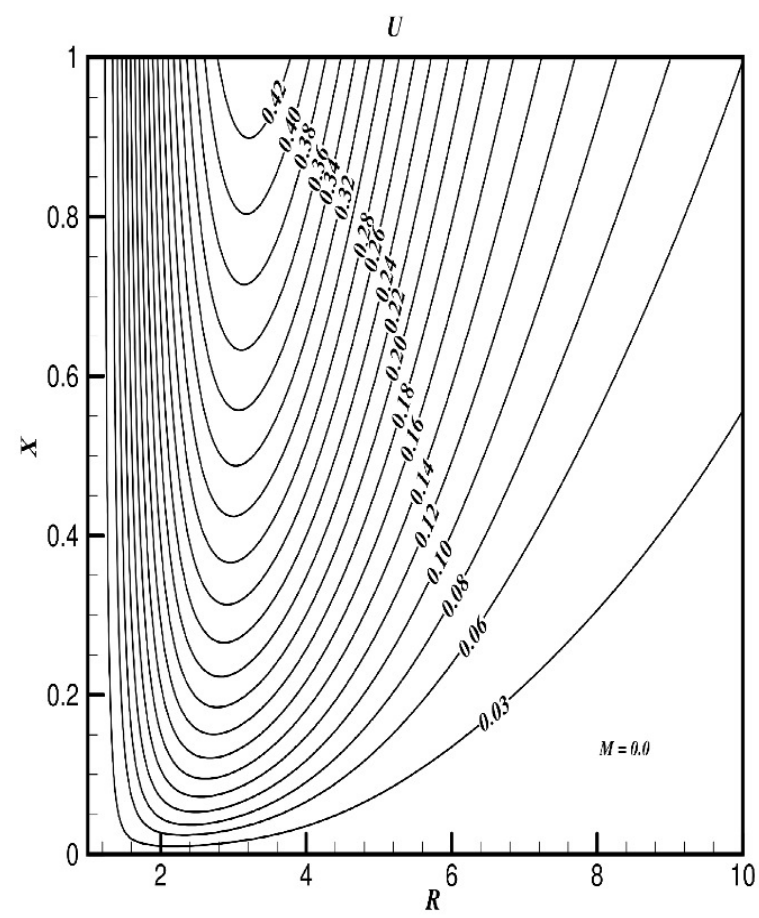

(a)

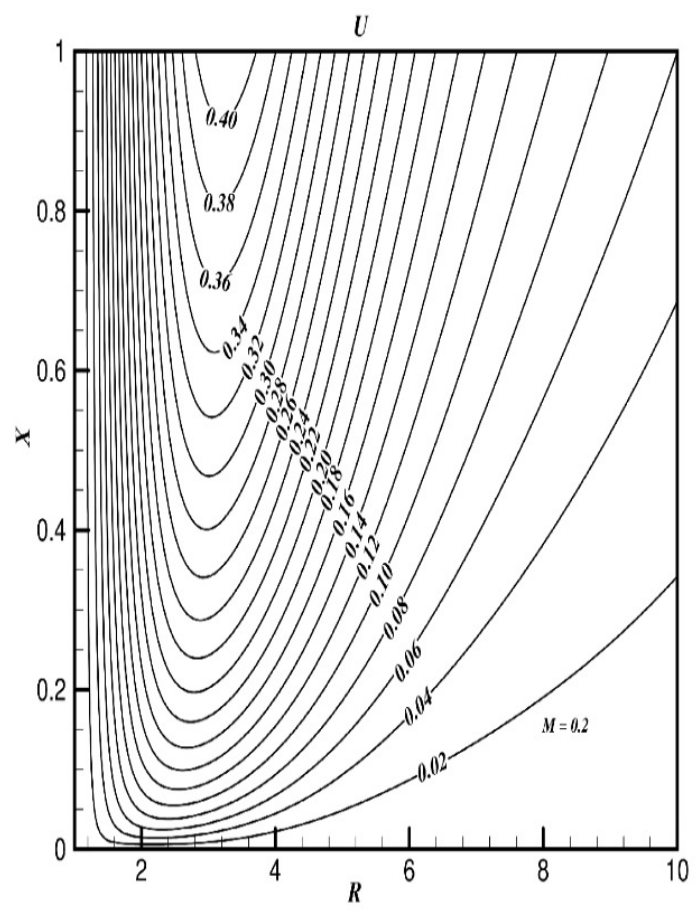

(b)

Figure 16: Steady state velocity contours (a) $M=0.0(b) M=0.2$ for fixed $S c=0.6, P r=0.7, B u=1.0, K r=-0.2, N r=2.0, K 1=1.0$, $E c=0.1$.

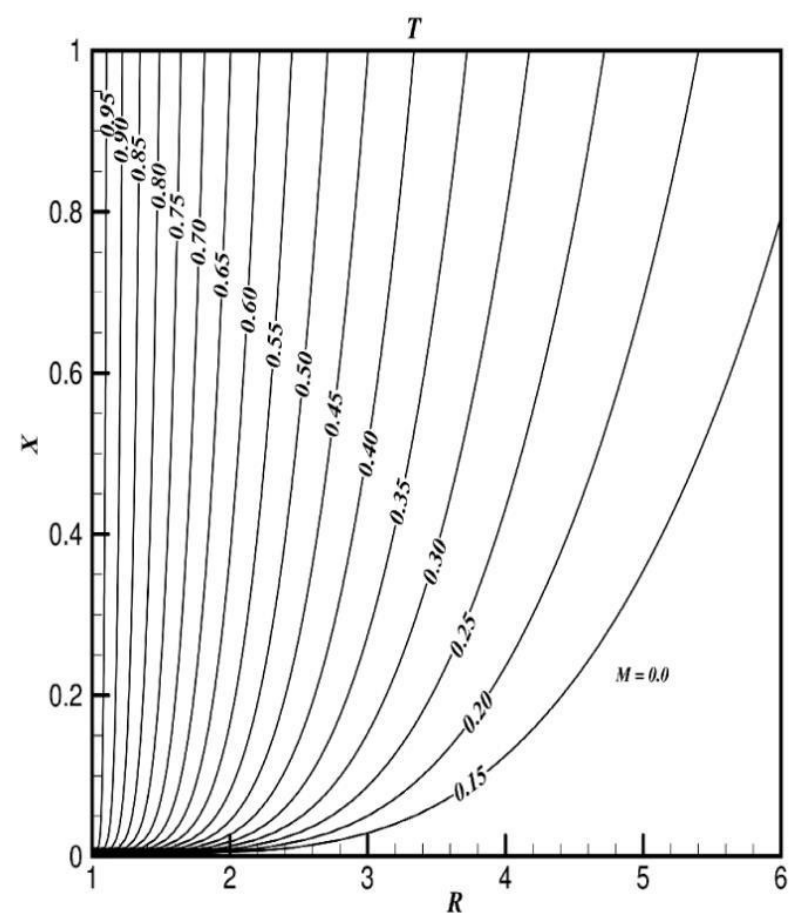

(a)

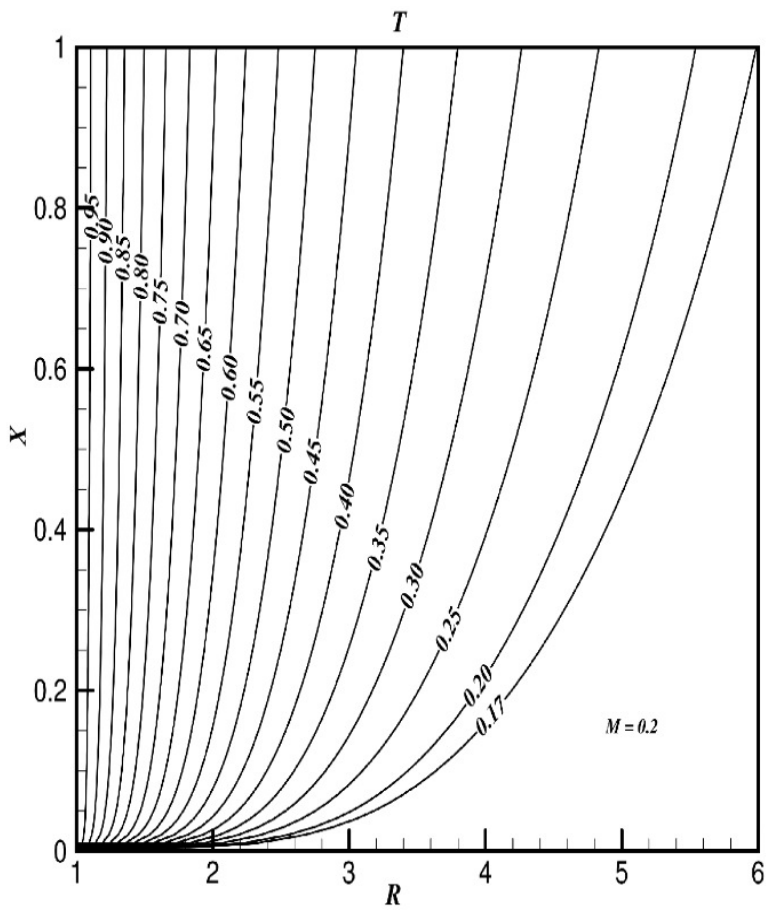

(b)

Figure 17: Steady state temperature contours (a) $M=0.0(b) M=$ 0.2 for fixed $S c=0.6, P r=0.7, B u=1.0, K r=-0.2, N r=2.0$, $K 1=1.0, E c=0.1$. 


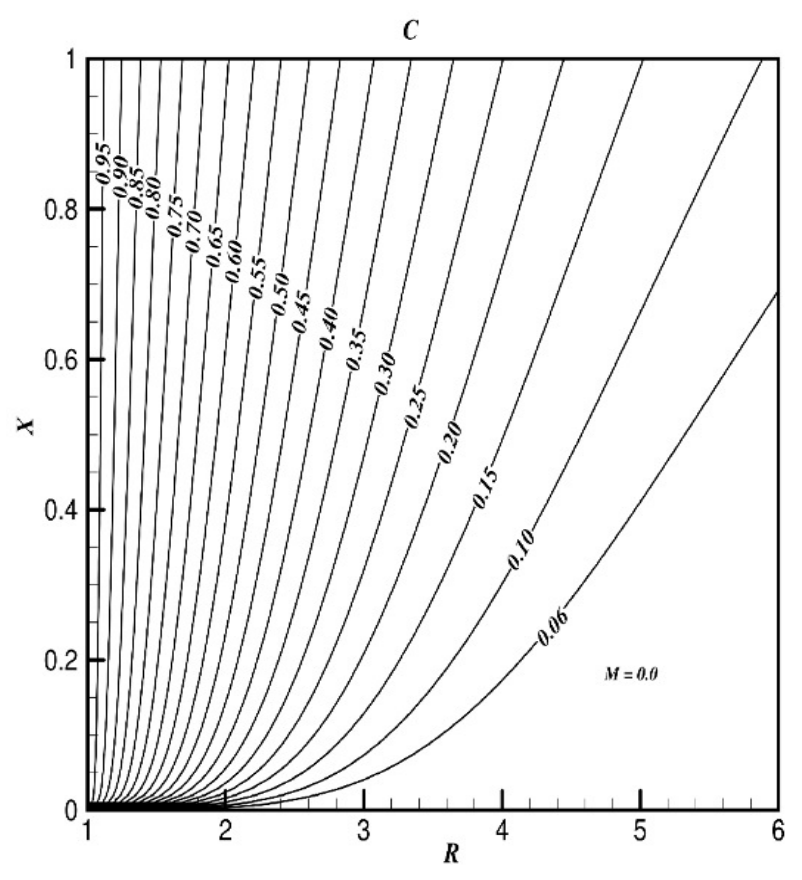

(a)

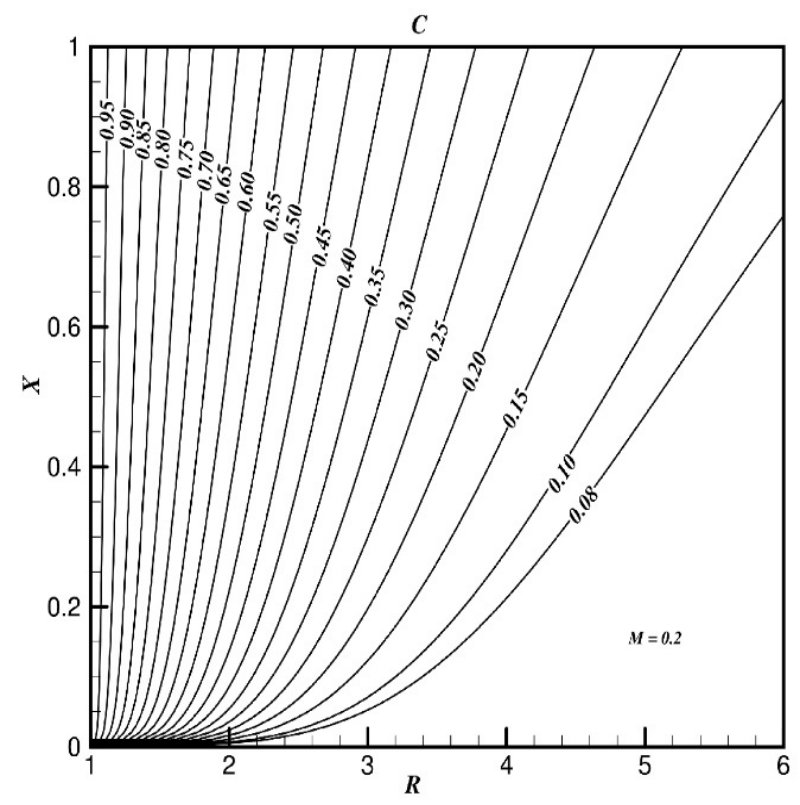

(b)

Figure 18: Steady state concentration contours $(a) M=0.0(b)$ $M=0.2$ for fixed $S c=0.6, P r=0.7, B u=1.0, K r=-0.2$, $N r=2.0, K 1=1.0, E c=0.1$.

for different values of $K 1$. The temperature profile starts at the hot cylindrical wall with $T=1$ and then for all time diminished to zero in $R$-direction monotonically. Steady state temperature profile decayed with enhancing $K 1$.

\section{(iii) Influence of radiation parameter $(\mathrm{Nr})$ on flow} profiles

Velocity profile: Figure 8(a) disclose the simulated transient velocity profile for distinct $\mathrm{Nr}$ values at position $(1,1.3)$. It is observed that, rising $N r$ values raises the flow field. It is because of the reason that, the bond holding tendency of components of the fluid particles are easily broken when the intensity of heat produced through convicted heat is raised, hence by increasing the thermal radiation parameter, the translational velocity increases. From this figure it is noticed that, the transient velocity increases gradually, reached maxima as well as minima and slightly increases then shortly later attains the steady state.

Figure $8(\mathrm{~b})$ describes the computer generated flow field at $X=1$ against $R$ for distinct $N r$. Initially all velocity curves are originated from the zero value and are merged with each other, after some distance they separate with each other and attains their maximum and gradually decreases to zero. Steady state flow field magnifies with rising $\mathrm{Nr}$.

Temperature profile: The Figure 9(a) provides the simulated time-dependent temperature profile for distinct $N r$ values at position $(1,1.05)$. The parameter $N r$ defines the comparative contribution of conduction heat transfer to convection heat transfer. It is seen from Figure 9(a) that, the rising value of $\mathrm{Nr}$ makes in increase in the temperature profile. It is because of fact that, the increasing $\mathrm{Nr}$ enhances the heat generation process and hence increases the thermal boundary layer thickness. Also, it is perceived that, initially all the thermal curves are coinciding with each other, later gradually increases and scatter with respect to time, attain slightly temporal maxima as well as minima, and then increases slowly there after reaches the steady state. The time required to attain the steady state decays with rising values of $\mathrm{Nr}$.

Steady state temperature profile for varying values of $N r$ is shown in the Figure 9(b) at $X=1$ against $R$. Also, the temperature profile generated from the heated cylinder wall at $T=1$ and diminished to zero in radial path irrespective of $t$. Further, the steady state thermal field amplifies with enhancing $\mathrm{Nr}$.

Concentration profile: Figure 10(a) shows the timedependent mass diffusion profile at the location (1, 1.06) for various values of $\mathrm{Nr}$. It is inspected that, the concentration field decayed for rising values of $\mathrm{Nr}$. Since, the thermal radiation process produces the heat by increasing $\mathrm{Nr}$ consequently the concentration field decreases by rising the $N r$. Further, the Figure 10(b) disclose the timeindependent mass diffusion field for various values of $\mathrm{Nr}$ 
at $X=1$ verses $R$. The concentration profile begins from the hot wall of the cylinder at $C=1$ and diminished to zero in radial path. Also, the steady state concentration profile decreases with increasing $\mathrm{Nr}$ values.

(iv) Influence of chemical reaction parameter (Kr) on velocity and concentration profiles

Velocity profile: Figure 11(a) indicates the impact of $K r$ on velocity profile at location $(1,1.3)$. Initially all velocity curves rising with respect to time, attains its maximum, then declines, again enhances and there after accomplishes the stable state. Initially when $t \ll 1$, the thermal flow is because of conduction effect. Immediately after sometimes, there exists a convective heat transfer process and which dominates the conduction phenomena. It is observed that for rising the values of $K r$, yields the diminished mass diffusion process, which in turns decays the values of $B u C$ in Eq.(12), due to this fact, the acceleration reduces. Hence, velocity profile diminished for the rising values of $K r$.

Figure 11(b) discloses the time-independent behaviour of simulated velocity field verses $R$ at $X=1$, at which flow curves begins with zero at cylinder, attains their highest value, then monotonically decayed to zero irrespective of time in $R$-path. Magnitude of the velocity field decreases with rising $K r$, since rising $K r$ results the reduced mass diffusion process near the cylinder surface (ref. Eq. 14), and this fact decelerate the flow field (ref. Eq. (12)). Hence, steady-state velocity diminished with rising $K r$.

Concentration profile: Figure 12(a) shows the effect of $\mathrm{Kr}$ on transient simulated concentration field at the location $(1,1.06)$. Where the concentration profiles initially near the wall dominated by conduction process, later attains temporal maxima as well as minima, then marginally enhances and shortly later touches to time-independent state. Temporal maxima attains early for smaller $K r$ values. Species diffusion field declined with amplifying $K r$, since higher values of $K r$ decreases the mass diffusion process in the flow domain (ref. Eq. (14)).

Simulated time-independent mass diffusion profile for various values of $K r$ at $X=1$ along $R$ path is described in Figure 12(b). The mass diffusion profile begins from the heated cylinder surface at $C=1$ and monotonically diminished to zero. Further, as $\mathrm{Kr}$ rises, the species concentration profile decreases.

(v) Effect of buoyancy ratio parameter $(\mathrm{Bu})$ on velocity profiles

Figures 13(a) and 13(b) describes the influence of buoyancy ratio parameter on velocity field. It is recorded from these figures that, the both transient and steady velocity profiles increases with increasing values of $B u$, this is due to the fact that, the increasing $B u$ enhances buoy- ancy forces and which in turn accelerates the flow field in the flow regime.

(vi) Effect of Eckert number (Ec) on temperature profiles

Figures 14(a) and 14(b) describes the influence of Eckert number on thermal field. It is recorded from these figures that, the both transient and steady temperature fields enhanced with rising values of $E c$, this is due to the fact that, the increasing $E c$ enhances frictional forces and which in turn accelerates the flow field in the flow regime.The physical justification behind this fact is that the presence of frictional forces in the fluid causes the release of heat energy into the fluid which gives the intensified temperature field in the flow region.

\subsection{Influence of magnetic parameter on physical quantities of interest}

Figure 15(a) reveals impact of magnetic parameter on transient average $\bar{C}_{f}$. This figure discloses that, enhancing magnetic variable diminished the skin-friction coefficient in the flow domain. Figure 15(b) describes the action of $M$ on average Nusselt number $(\overline{N u})$ and it is known that, $\overline{N u}$ is the ratio of heat transfer due to convection to the heat transfer due to conduction, if its value is one, it reflects the thermal transfer by pure conduction. Figure 15(b) discloses that, amplifying $M$ decays the $\overline{N u}$ in the flow domain. Further, the Figure 15(c), displays the effect of Mon average Sherwood number $(\overline{S h})$. However, $\overline{S h}$ is the ratio of convective mass transfer to the diffusive mass transport process. It is recorded from Figure 15(c) that, the rising $M$ diminishes the $\overline{S h}$ in the flow domain.

\subsection{Contours of the flow fields}

Figures 16(a) and 16(b) disclose the impact of $M$ on the steady-state velocity contours in the two-dimensional domain. Figures 16(a) and 16(b) demonstrate that, velocity field of couple stress fluid for $M=0.0$ is higher when compared to $M=0.2$, it is due to Lorentz's effect. Further, it is observed that, as $M$ increases, the velocity contours of couple stress fluid moves away from the heated cylindrical surface. Figures 17(a) and 17(b) describe the stable state thermal contours for different $M$ values. Thermal field of couple stress fluid for $M=0.0$ is less when equated to $M=0.2$. Further, for the enhancing $M$, the thermal contours moves near to heated cylinder. Figure 18(a) and 18(b) shows the steady-state concentration contours for differ- 
ent values of $M$. It is noticed that, as $M$ increases, mass diffusion contours of couple stress fluid moves far from the heated cylindrical wall.

\section{Concluding remarks}

In the current investigation, authors obtained the simulated results on the boundary layer flow of viscous incompressible non-Newtonian unsteady magnetized thermally radiative couple stress fluid flow about a cylinder with viscous dissipation effect under the influence of porous medium and chemical reaction. The system of governing nonlinear non-dimensional equations with required conditions are simplified by employing the implicit FDM. Solutions are obtained for different values of magnetic field, porous medium parameter, thermal radiation parameter and chemical reaction parameter. From the present study following observations are made in limiting sense:

- Velocity profile decreases, whereas temperature and concentration profiles increase for amplifying $M$.

- Velocity field increases and temperature profile decreases with rising $K 1$ values.

- Velocity and temperature profiles increase whereas concentration profile decreased with enhancing values of $N r$.

- Velocity and concentration profiles decreased with rising values of $K r$.

- Momentum, thermal and species transport rates decreased for amplifying values of $M$.

- Velocity profile increases with increase buoyancy ratio parameter.

- Temperature enhanced with rising Eckert number.

- Time-independent state flow and mass diffusion contours deviates away from the cylinder surface, whereas, the temperature contours lies near to heated cylinder for the rising $M$.

However, it is anticipated that, the present numerical investigation provides the motivation for numerical modelling of two and three dimensional heat and mass transfer problems occurring in the field of science and engineering. Further, the present paper gives a motivation for the modeling of couple stress fluid flow problems past a horizontal cylinder, sphere, cone and stretching cylinder as well as sheets with various physical aspects. Also, the present problem can be extended to study the problems occurring in the field of bioengineering and medicine, solar collectors, nonmaterial synthesis, thermal and chemical industries, etc.

Acknowledgements: The authors wish to express their gratitude to the reviewers who highlighted important areas for improvement in the earlier draft of this article. Their suggestions have served specifically to enhance the clarity and depth of the interpretation of results in the revised manuscript.

Funding information: The first author wishes to thank Government of India, Ministry of Education (MoE), University Grant Commission (UGC), for the grant of research fellowship ((Reg. No: 400170), (UGC Ref. No: 956/ (CSIR-NET JUNE-2018)) and to the Central University of Karnataka for providing the research facilities.

Author contributions: All authors have accepted responsibility for the entire content of this manuscript and approved its submission.

Conflict of interest: The authors state no conflict of interest.

\section{List of symbols:}

$u$ - velocity along the vertical axis

$v$ - velocity along the radial direction

$t^{\prime}$ - time

$t$ - non-dimensional time

$T^{\prime}$ - temperature

$T$ - dimensionless temperature

$C^{\prime}$ - concentration

$C$ - dimensionless concentration

$K_{1}^{\prime}$ - permeability parameter of the medium

K1- dimensionless Porous parameter

Ec- Eckert number

$q_{r}-$ radiative heat flux

$K_{r}^{\prime}$ - Coefficient of chemical reaction

$\mathrm{Kr}$ - Dimensionless chemical reaction parameter

$B_{0}$ - Magnetic field

g- Gravitational field

$\mathrm{Gr}_{T}, \mathrm{Gr}_{\mathrm{C}^{-}}$thermal and solute Grashof numbers

$M$ - magnetic number

$B u$ - combined buoyancy ratio parameter

$r_{0}-$ radius of the cylinder

$S c$ - Schmidt number

$\mathrm{Pr}$ - Prandtl number

$\mathrm{Nr}$ - thermal radiation parameter

$k$ - thermal conductivity

$r$ - radial coordinate

$x$ - axial coordinate

$U, V$ - dimensionless velocities 
$X$ - non-dimensional axial coordinate

$R$ - non-dimensional radial coordinate

$\nabla^{4}$ - Biharmonic or Bi-Laplacian operator

\section{Greek symbols:}

$\alpha$ - thermal diffusive coefficient

$\beta_{T}$ - thermal expansion coefficient

$\beta_{C^{-}}$mass diffusion expansion coefficient

$\mu$-dynamic viscosity

$v$ - kinematic viscosity

$\rho$ - density

$\sigma$ - electrical conductivity of the fluid

$\eta$ - material constant

\section{Subscripts:}

$w$ - wall condition

$\infty-$ ambient condition

\section{Superscripts:}

$n$ - time step level

\section{References}

[1] Soundalgekar VM. Effects of mass transfer and free convection currents on the flow past an impulsively started vertical plate. J Appl Mech. 1979;46(4):757-760.

[2] Soundalgekar VM, Murty TV. Heat transfer in flow past a continuous moving plate with variable temperature. Heat Mass Transf. 1980;14:91-93.

[3] Soundalgekar VM, Takher HS. Dissipation effects on MHD free convection flow past a semi-infinite vertical plate. Appl Sci Res. 1980;36:163-171.

[4] Ganesan P, Rani HP. Transient natural convection along vertical cylinder with heat and mass transfer. Heat Mass Transf. 1998;33:449-455.

[5] Ganesan P, Rani HP. Unsteady free convection MHD flow past a vertical cylinder with heat and mass transfer. Int J Therm Sci. 2000;39:265-272.

[6] Fathia M, Samman AL, Gokhale MY, Soundalgekar VM. Transient free convective flow of a viscous dissipative fluid with mass transfer past a semi-infinite vertical plate. Indian J. Eng. Mater. Sci. 2002;9:49-57.

[7] Rani HP. Transient natural convection along a vertical cylinder with variable surface temperature and mass diffusion. Heat Mass Transf. 2003;40:67-73.

[8] Singh AK, Soundalgekar VM, Dekha R. Transient MHD free convection viscous dissipative fluid past an infinite vertical plate. J Rajastan Acad Phys Sci. 2006;5:239-250.

[9] Palani G. Viscous dissipative fluid flow past a semi-infinite vertical plate with variable surface temperature. Int J Numer Meth Fluid. 2007;55:1157-1169.

[10] Rani HP, Kim KY. Transient convection on a vertical cylinder with variable viscosity and thermal conductivity. J Thermophys
Heat Trans. 2008;22(2):254-261.

[11] Rani HP, Kim KY. Transient free convection flow over an isothermal vertical cylinder with temperature dependent viscosity. Korean J Chem Eng. 2008;25(1):34-40.

[12] Rani HP, Kim KY. A numerical study of the Dufour and Soret effects on unsteady natural convection flow past an isothermal vertical cylinder. Korean J Chem Eng. 2009;26(4):946-954.

[13] Palani G, Srikanth U. MHD flow past a semi-infinite vertical plate with mass transfer. Nonlin Anal Model Control. 2009;14:345-356.

[14] Palani G, Kim KY. Numerical study on a vertical plate with variable viscosity and thermal conductivity. Arch Appl Mech. 2010;80:711-725.

[15] Rani HP, Kim KY. A transient natural convection of micro polar fluids over a vertical cylinder. Heat Mass Transf. 2010;46:1277-1285.

[16] Rani HP, Reddy GJ, Kim CN. Numerical analysis of couple stress fluid past an infinite vertical cylinder. Eng Appl Comput Fluid Mech. 2011;5(2):159-169.

[17] Rani HP, Reddy GJ, Kim CN. Transient analysis of diffusive chemically reactive species for couple stress fluid flow past a vertical cylinder. Appl Math Mech. 2013;34(8):985-1000.

[18] Loganathan P, Kannan V, Ganesan P. Thermal radiation effects on MHD flow over a moving semi-infinite vertical cylinder. Int J Math Anal. 2011;5:257-274

[19] Neog BC, Das R. Unsteady free convection MHD flow past a vertical plate with variable temperature and chemical reaction. Int J Eng Res Tech. 2012;1(5):1-5.

[20] Rani HP, Reddy GJ, Kim KY. The effect of the couple stress parameter and Prandtl number on the transient natural convection flow over a vertical cylinder. Acta Mech Sin. 2013;29(5):649-656.

[21] Bella CS, Naikoti K. Radiation effects on unsteady MHD convective heat and mass transfer past a vertical plate with chemical reaction and viscous dissipation. Alexandria Eng J. 2015;54(3):661-671.

[22] Hossain MA, Mondal RK, Ahmed R, Ahmmed SF. A numerical study on unsteady natural convection flow with temperature dependent viscosity past an isothermal vertical cylinder. Indian J Pure Appl Phys. 2015;5(5):125-135.

[23] Loganathan P, Sivapoornapriya C. unsteady natural convective flow over an impulsively started semi-infinite vertical plate in the presence of porous medium with chemical reaction. J Appl Fluid Mech. 2016;9:95-102.

[24] Reddy DB, Raju GSS, Radiation and chemical reaction effects on unsteady MHD mixed convection flow over a radiative vertical porous plate with radiation absorption. Glob. J Pure Appl Math. 2017;13(8):4015-4034.

[25] Palani G, Srikanth U, Kim CN. Combined effects of viscous dissipation and MHD on free convection flow past a semiinfinite vertical plate with variable surface temperature in the presence of heat source. J Eng Thermophys. 2017;26:113-124.

[26] Loganathan P, Divya M. Numerical investigation on viscous dissipating and chemically reactive fluid over an impulsively started vertical cylinder. Indian J Pure Appl Phys. 2018;56:551560.

[27] Reddy BP. Radiation and chemical reaction effects on unsteady MHD free convection parabolic flow past an infinite isothermal vertical plate with viscous dissipation. Int J Appl Mech Eng. 2019;24:343-358. 
[28] Basha H, Reddy GJ, Narayanan NSV. A numerical study of natural convection properties of supercritical water using RedlichKwong equation of state. Sādhanā. 2019;44(37):1-15.

[29] Basha H, Reddy GJ, Reddy MG. Chemically reactive species of time-dependent natural convection couple stress fluid flow past an isothermal vertical flat plate. Can J Phys. 2019;97(2):166-175.

[30] Shekar BC, Kishan N. Finite element analysis of natural convective heat transfers in a porous square cavity filled with Nanofluids in the presence of thermal radiation. J Phys Conf Ser. 2015;662(1):1-13.

[31] Haritha C, Shekar BC, Kishan N. MHD natural convection heat transfer in a porous square cavity filled by nanofluids with viscous dissipation. J Nanofluids. 2018;7:928-938.

[32] Balla CS, Alluguvelli R, Naikoti K, Makinde OD. Effect of chemical reaction on bioconvective flow in oxytactic microorganisms suspended porous cavity. J Appl Comput Mech. 2020;6(3):653664.

[33] Acharya N, Mondal H, Kundu PK. Spectral approach to study the entropy generation of radiative mixed convective couple stress fluid flow over a permeable stretching cylinder. Proc Inst Mech Eng C J Mech Eng Sci. 2020;235(15):2692-2704.

[34] Basha H, Reddy GJ, Narayanan NSV, Sheremet MA. Analysis of supercritical free convection in Newtonian and couple stress fluids through EOS approach. Int J Heat Mass Transf. 2020;152:119542.

[35] Das S, Patra RR, Jana RN. The layout of Boussinesq couplestress fluid flow over an exponentially stretching sheet with slip in porous space subject to a variable magnetic field. Multidiscip Model Mater Struct. 2020;5:1131-1154.

[36] Ibrahim W, Gadisa G. Double stratified mixed convective flow of couple stress nanofluid past inclined stretching cylinder using Cattaneo-Christov heat and mass flux model. Adv Math Phys. 2020;2020:1-16.

[37] Ramudu ACV, Kumar KA, Sugunamma V, Sandeep N. Heat and mass transfer in MHD Casson nanofluid flow past a stretching sheet with thermophoresis and Brownian motion. Heat Transf. 2020;49(8):5020-5037.

[38] Hassan AR, Disu AB, Fenuga 0J. Entropy generation effect of a buoyancy force on hydromagnetic heat generating couple stress fluid through a porous medium with isothermal boundaries. Heliyon. 2020;6:e04156.

[39] Mabood F, Yusuf TA, Bognar G. Features of entropy optimization on MHD couple stress nanofluid slip flow with melting heat transfer and nonlinear thermal radiation. Sci Rep. 2020;10:19163-13.

[40] Sadiq MN, Sajid M, Javed T, Ali N. A numerical study for heat and fluid flow of couple stress fluid over a spiraling disk by Legendre wavelet spectral collocation method. Z Angew Math Mech. 2020;100(8):1-13.

[41] Brewster MQ. Thermal radiative transfer and properties. John Wiley \& Sons, New York, USA; 1992. 